\title{
Limits from BBN on light electromagnetic decays
}

\author{
Lindsay Forestell, ${ }^{a, b}$ David E. Morrissey ${ }^{b}$ and Graham White ${ }^{b}$ \\ ${ }^{a}$ Department of Physics and Astronomy, University of British Columbia, \\ Vancouver, BC V6T 1Z1, Canada \\ ${ }^{b}$ TRIUMF, 4004 Wesbrook Mall, \\ Vancouver, BC V6T 2A3, Canada \\ E-mail: Imforest@phas.ubc.ca, dmorri@triumf.ca, gwhite@triumf.ca
}

ABSTRACT: Injection of electromagnetic energy — photons, electrons, or positrons - into the plasma of the early universe can destroy light elements created by primordial Big Bang Nucleosynthesis (BBN). The success of BBN at predicting primordial abundances has thus been used to impose stringent constraints on decay or annihilation processes with primary energies near or above the electroweak scale. In this work we investigate the constraints from $\mathrm{BBN}$ on electromagnetic decays that inject lower energies, between $1-100 \mathrm{MeV}$. We compute the electromagnetic cascade from such injections and we show that it can deviate significantly from the universal spectrum commonly used in BBN calculations. For electron injection below $100 \mathrm{MeV}$, we find that the final state radiation of photons can have a significant impact on the resulting spectrum relevant for BBN. We also apply our results on electromagnetic cascades to investigate the limits from $\mathrm{BBN}$ on light electromagnetic decays prior to recombination, and we compare them to other bounds on such decays.

Keywords: Beyond Standard Model, Cosmology of Theories beyond the SM

ARXiv EPrint: 1809.01179 


\section{Contents}

1 Introduction $\quad 2$

2 Development of the electromagnetic cascade 4

2.1 Computing the electromagnetic cascade 4

2.1.1 Monochromatic photon injection 5

$\begin{array}{ll}\text { 2.1.2 Monochromatic electron injection } & 7\end{array}$

2.2 Review of the universal spectrum 8

2.3 Results for photon injection $\quad 9$

$\begin{array}{lll}2.4 & \text { Results for electron injection } & 10\end{array}$

3 Effects of electromagnetic injection on BBN 11

$\begin{array}{ll}3.1 \text { Photodissociation of light elements } & 11\end{array}$

$\begin{array}{lll}3.2 & \text { BBN constraints on photon injection } & 13\end{array}$

$\begin{array}{lll}3.3 & \mathrm{BBN} \text { constraints on electron injection } & 13\end{array}$

4 Other constraints on electromagnetic decays $\quad 15$

$\begin{array}{lll}5 & \text { Conclusions } & 17\end{array}$

$\begin{array}{lr}\text { A Relaxation and transfer rates } & \mathbf{1 8}\end{array}$

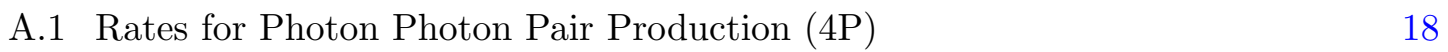

$\begin{array}{ll}\text { A.1.1 4P photon relaxation } & 19\end{array}$

$\begin{array}{lll}\text { A.1.2 4P transfer to electrons } & 19\end{array}$

A.2 Rates for Inverse Compton scattering (IC) 20

A.2.1 IC electron relaxation 20

A.2.2 IC transfer to electrons 20

A.2.3 IC transfer to photons 21

A.3 Photon Photon Scattering (PP) 21

$\begin{array}{lll}\text { A.3.1 PP photon relaxation } & 21\end{array}$

$\begin{array}{lll}\text { A.3.2 PP photon transfer } & 21\end{array}$

$\begin{array}{lll}\text { A.4 Pair Creation on Nuclei (PCN) } & 21\end{array}$

$\begin{array}{lll}\text { A.4.1 PCN photon relaxation } & 21\end{array}$

A.4.2 PCN electron transfer $\quad 22$

A.5 Compton Scattering (CS) 23

A.5.1 CS photon relaxation 23

$\begin{array}{lll}\text { A.5.2 CS photon transfer } & 23\end{array}$

$\begin{array}{lll}\text { A.5.3 CS electron transfer } & 23\end{array}$ 


\section{Introduction}

Big Bang Nucleosynthesis (BBN) is one of the most powerful probes of the very early universe [1-4]. Over the course of BBN, free protons and neutrons assemble into a handful of light elements [5-7]. Assuming a standard $\Lambda \mathrm{CDM}$ cosmological history, the primordial abundances of these elements can be predicted using known nuclear reaction rates in terms of a single input parameter, the overall baryon density. These predictions agree well with observational determinations of primordial abundances up to plausible uncertainties in astrophysical determinations and nuclear rates [8]. ${ }^{1}$

The success of BBN gives very strong evidence for the $\Lambda \mathrm{CDM}$ cosmological model up to radiation temperatures near the $\mathrm{MeV}$ scale [13-15], which extends much earlier than other known tests [16]. BBN also places stringent constraints on new physics beyond the Standard Model that injects energy into the cosmological plasma or influences the expansion rate at early times. This includes the decays of massive particles with lifetimes greater than $\tau \simeq 0.1 \mathrm{~s}$ [17-30], dark matter (DM) annihilation with an effective cross section near the critical value for thermal freeze-out [31-34], and any new thermalized species with mass below a few $\mathrm{MeV}$ [35-38].

Limits from BBN on the decays of long-lived massive particles have been studied in great detail [26-30]. In the majority of this work, often motivated by new physics connected to the electroweak hierarchy puzzle or weakly-interacting massive particle (WIMP) dark matter, the energy injected by the decay has been assumed to be close to or greater than the weak scale. Thus, the decay products typically have initial energies that are much larger than the thresholds for nuclear reactions relevant to BBN which are typically on the order of several $\mathrm{MeV}$. Weak-scale decay products typically also have both hadronic and electromagnetic components, if only through radiative effects.

Hadronic energy injection can modify the light element abundances at times as early as $t \sim 0.1 \mathrm{~s}[26,27]$. Initially, these products scatter with protons and neutrons and alter the ratio of these baryons and thus the resulting helium abundance. At later times, injected hadrons destroy and modify the abundances of helium and other light elements through hadrodissociation. Since the initial hadronic energies are usually assumed to be much larger than the MeV scale, thresholds for these reactions are easily overcome.

Electromagnetic energy - photons, electrons, and positrons - injected into the cosmological plasma does not have a significant effect on the light element abundances until much later. The main effect of electromagnetic injection on the light elements is photodissociation (unless the amount of energy deposited is enormous). However, being much lighter than hadrons, photons and electrons lose their energy very efficiently by scattering off the highly-abundant photon background. The electromagnetic cascade initiated by this scattering is strongly suppressed for energies above $E_{c}$, given by $[39,40]$

$$
E_{c} \simeq \frac{m_{e}^{2}}{22 T} \simeq(2 \mathrm{MeV})\left(\frac{6 \mathrm{keV}}{T}\right)
$$

\footnotetext{
${ }^{1}$ The extrapolated densities of ${ }^{6} \mathrm{Li}$ and ${ }^{7} \mathrm{Li}$ give a particularly acute puzzle in this regard [9-12].
} 
where $T$ is the cosmological photon temperature. As a result, even for initial energies orders of magnitude above the $\mathrm{MeV}$-scale thresholds for photodissociation, the fraction of energy available for photodissociation is tiny until the background temperature falls below $T \lesssim 10 \mathrm{keV}$, corresponding to $t \sim 10^{4} \mathrm{~s}$.

While much of the focus on new sources of energy injection during BBN has been on decays or annihilations at or above the weak scale, there exist many well-motivated theories that also predict new sources well below the weak scale. Specific examples include dark photons [41, 42], dark Higgs bosons [42, 43], dark gluons and glueballs [44-46], light or strongly-interacting dark matter $[47,48]$, and $\mathrm{MeV}$-scale neutrino decays $[49,50]$. As the injection energy falls below the GeV scale, hadronic decay channels start to become kinematically unavailable and disappear entirely below the pion threshold. This leaves electromagnetic and neutrino injection as the only remaining possibilities. Even more importantly, it was shown in refs. $[51,52]$ that the development of the electromagnetic cascade at these lower energies can differ significantly relative to injection above the weak scale. Furthermore, as the injection energy falls below a few tens of $\mathrm{MeV}$, photodissociation reactions begin to shut off.

In this work we expand upon the analysis of refs. [51, 52] and investigate the effects of electromagnetic energy injection below $100 \mathrm{MeV}$ on the primordial element abundances created during BBN. A major focus of this study is the development of the electromagnetic cascade from initial photon or electron $\left(e^{+} e^{-}\right)$injection. For high energy injection, the resulting spectrum of photons is described very well by the so-called universal spectrum rescaled by a temperature- and energy-dependent relaxation rate. This spectrum is used widely in studies of photodissociation effects on BBN, it can be parametrized in a simple and convenient way, and has the attractive feature that it only depends on the total amount of electromagnetic energy injected. However, for lower-energy electromagnetic injection, the universal spectrum does not properly describe the resulting electromagnetic cascades.

The universal spectrum fails for lower-energy injection in two significant ways. First, the universal spectrum is based on a fast redistribution of the initial energy $E_{X} \gg E_{c}$ to a spectrum populated at $E \leq E_{c}$ through Compton scattering and photon-photon pair production. As shown in refs. $[51,52]$, this picture does not hold for initial injection energies $E_{X}<E_{c}$, which can easily occur for smaller $E_{X}$ and larger decay lifetimes. And second, as argued in ref. [42] the Compton scattering with background photons that dominates electron interactions is qualitatively different at high energies compared to low. At higher energies, $s \sim E T \gg m_{e}^{2}$, electrons scatter in the Klein-Nishina limit and typically lose an order unity fraction of their energy in each scattering event. In contrast, lower energy scattering with $s \sim E T \ll m_{e}^{2}$ enters the Thomson regime where the fractional change in the electron energy per collision is very small and the up-scattered photon energy is much less than the initial electron energy.

To address the breakdown of the universal spectrum for lower-energy electromagnetic injection, we compute the full electromagnetic cascade for photon or electron $\left(e^{+} e^{-}\right)$injection with initial energies $E_{X} \in[1,100] \mathrm{MeV}$ following the methods of ref. [40]. Our work expands upon refs. $[51,52]$ that studied photon portion of the cascade for photon injection. We compare and contrast our results to the universal spectrum, and study their implica- 
tions for BBN. In addition to finding important differences from the universal spectrum at these lower energies, we also demonstrate that final-state radiation (FSR) from electron injection can have a very significant impact on the resulting photon spectrum. For very low injection energies approaching the $\mathrm{MeV}$ scale, we also study the interplay of the spectrum with the thresholds for the most important nuclear photodissociation reactions.

The outline of this paper is as follows. After this introduction, we present our calculation of the electromagnetic cascade in section 2. Next, in section 3 we study the impact of such electromagnetic injection on the light element abundances. In section 4 we contrast the bounds from photodissociation of light elements with other limits on late electromagnetic injection. Finally, section 5 is reserved for our conclusions. Some technical details are listed in appendix A for completeness.

\section{Development of the electromagnetic cascade}

In this section we compute the electromagnetic cascade in the early universe following the injection of photons or electrons $\left(e^{+} e^{-}\right)$with initial energy $E_{X}<100 \mathrm{MeV}$.

\subsection{Computing the electromagnetic cascade}

Energetic photons or electrons injected into the cosmological plasma at temperatures below the $\mathrm{MeV}$ scale interact with background photons and charged particles leading to electromagnetic cascades that produce spectra of photons and electrons at lower energies. Since the development of the cascade is much faster than the typical interaction time with the much more dilute light elements created in BBN, these spectra can be used as inputs for the calculation of photodissociation effects.

The most important reactions for the development of the electromagnetic cascade in the temperature range of interest $T \in[1 \mathrm{eV}, 10 \mathrm{keV}]$ are [40]:

- photon photon pair production (4P): $\gamma+\gamma_{\mathrm{BG}} \rightarrow e^{+}+e^{-}$

- photon photon scattering (PP): $\gamma+\gamma_{\mathrm{BG}} \rightarrow \gamma+\gamma$

- pair creation on nuclei (PCN): $\gamma+N_{\mathrm{BG}} \rightarrow N_{\mathrm{BG}}+e^{+}+e^{-}$

- Compton scattering (CS): $\gamma+e_{\mathrm{BG}}^{-} \rightarrow \gamma+e^{-}$

- inverse Compton (IC): $e^{\mp}+\gamma_{\mathrm{BG}} \rightarrow e^{\mp}+\gamma$

- final state radiation (FSR): $X \rightarrow e^{+}+e^{-}+\gamma$

Of these processes, IC and 4P are typically the fastest provided there is enough energy for them to occur.

We define $\mathcal{N}_{a}=d n_{a} / d E$ to be the differential number densities per unit energy of photons $(a=\gamma)$ and the sum of electrons and positrons $(a=e)$. The Boltzmann equations for the evolution of these spectra take the form

$$
\frac{d \mathcal{N}_{a}}{d t}(E)=-\Gamma_{a}(E) \mathcal{N}_{a}(E)+\mathcal{S}_{a}(E)
$$


where $\Gamma_{a}(E)$ is a relaxation rate at energy $E$, and $\mathcal{S}_{a}(E)$ describes all sources at this energy. Since the relaxation rates are typically much faster than the Hubble rate, the Hubble dilution term has been omitted. Furthermore, the relaxation rate is also much larger than the mean photodissociation rates with light nuclei, so a further quasistatic approximation can made with $d \mathcal{N}_{a} / d t \rightarrow 0$ [40]. This gives the simple solution

$$
\mathcal{N}_{a}(E)=\frac{\mathcal{S}_{a}(E)}{\Gamma_{a}(E)} .
$$

Note that $\mathcal{N}_{a}(E)$ evolves in time in this approximation through the time and temperature dependences of the sources and relaxation rates. The source terms are discussed in more detail below while explicit expressions for the contributions to the relaxation rates are given in appendix A.

\subsubsection{Monochromatic photon injection}

For monochromatic photon injection at energy $E_{X}$ from a decay with rate per volume $R$, the source terms are

$$
\begin{aligned}
& S_{\gamma}(E)=\xi_{\gamma} R \delta\left(E-E_{X}\right)+\sum_{b} \int_{E}^{E_{X}} d E^{\prime} K_{\gamma b}\left(E, E^{\prime}\right) \mathcal{N}_{b}\left(E^{\prime}\right) \\
& \mathcal{S}_{e}(E)=0+\sum_{b} \int_{E}^{E_{X}} d E^{\prime} K_{e b}\left(E, E^{\prime}\right) \mathcal{N}_{b}\left(E^{\prime}\right)
\end{aligned}
$$

where $\xi_{\gamma}$ is the number of photons injected per decay, and the $K_{a b}\left(E, E^{\prime}\right)$ functions describe scattering processes that transfer energy from species $b$ at energy $E^{\prime}$ to species $a$ at energy $E \leq E^{\prime}$. Explicit expressions for these transfer functions are given in appendix A. Note that in the case of decays of species $X$ with lifetime $\tau_{X}$, the rate is $R=n_{X}(t) / \tau_{X}$. These equations can also be applied to annihilation reactions of the form $X+\bar{X} \rightarrow n \gamma$ with cross section $\langle\sigma v\rangle$ by setting $R=\langle\sigma v\rangle n_{X} n_{\bar{X}}$ and $\xi_{\gamma}=n$.

It is convenient to describe the cascades resulting from the initial monochromatic (delta function) injection with smooth functions that are independent of the injection rate. To this end, we define

$$
\begin{aligned}
& \bar{f}_{\gamma}(E)=\frac{1}{R} \mathcal{N}_{\gamma}(E)-\frac{\xi_{\gamma}}{\Gamma_{\gamma}\left(E_{X}\right)} \delta\left(E-E_{X}\right) \\
& \bar{f}_{e}(E)=\frac{1}{R} \mathcal{N}_{e}(E) .
\end{aligned}
$$

Using this form in eq. (2.2) with the sources of eqs. (2.3), (2.4), we obtain the relations

$$
\begin{aligned}
& \Gamma_{\gamma}(E) \bar{f}_{\gamma}(E)=\xi_{\gamma} \frac{K_{\gamma \gamma}\left(E, E_{X}\right)}{\Gamma_{\gamma}\left(E_{X}\right)}+\sum_{b} \int_{E}^{E_{X}} d E^{\prime} K_{\gamma b}\left(E, E^{\prime}\right) \bar{f}_{b}\left(E^{\prime}\right) \\
& \Gamma_{e}(E) \bar{f}_{e}(E)=\xi_{\gamma} \frac{K_{e \gamma}\left(E, E_{X}\right)}{\Gamma_{\gamma}\left(E_{X}\right)}+\sum_{b} \int_{E}^{E_{X}} d E^{\prime} K_{e b}\left(E, E^{\prime}\right) \bar{f}_{b}\left(E^{\prime}\right)
\end{aligned}
$$

The functions $\bar{f}_{\gamma}$ and $\bar{f}_{e}$ are expected to be smooth, and can be used to reconstruct the full spectra $\mathcal{N}_{\gamma}(E)$ and $\mathcal{N}_{e}(E)$ uniquely for any given injection rate $R$. 
Determining the electromagnetic cascade from monochromatic photon injection is therefore equivalent to solving eqs. (2.7), (2.8). We do so using the iterative method of ref. [40], with an important modification to account for the Thomson limit of IC scattering. In this method, the spectra $\bar{f}_{a}(E)$ are determined on a grid of energy points $E_{i}$ given by

$$
E_{i}=E_{0}\left(\frac{E_{N}}{E_{0}}\right)^{i / N}
$$

where we use $E_{0}=1 \mathrm{MeV}, E_{N}=E_{X}, i=0,1, \ldots, N$, and $N \gg 1$. For the top point $i=N$, eqs. (2.7), (2.8) give

$$
\bar{f}_{\gamma}\left(E_{N}\right)=\xi_{\gamma} K_{\gamma \gamma}\left(E_{N}, E_{N}\right) / \Gamma_{\gamma}^{2}\left(E_{N}\right), \quad \bar{f}_{e}\left(E_{N}\right)=\xi_{\gamma} K_{e \gamma}\left(E_{N}, E_{N}\right) / \Gamma_{\gamma}\left(E_{N}\right) \Gamma_{e}\left(E_{N}\right) .
$$

To compute the spectra at lower points, we use the fact that the transfer integrals at a given energy $E$ only depend on the spectra at energies $E^{\prime}>E$. Thus, at any step $i$ the integrals in eqs. (2.7), (2.8) can be approximated numerically (e.g. Simpson's rule) using the spectra already determined at points $j=i+1, \ldots, N$. Relative to ref. [40] we also apply a finer grid to compute the top two energy points.

This approach to computing the cascades works well for $y_{e}=E_{e} T / m_{e}^{2} \gg 1$, but becomes numerically challenging for $y_{e} \lesssim 0.1$. The problem comes from the contribution of inverse Compton (IC) scattering to $K_{e e}$. As $y_{e}$ becomes small, IC scattering enters the Thomson regime in which the cross section is large but the fractional change in the electron energy per scattering is much less than unity, and thus the function $K_{e e}\left(E, E^{\prime}\right)$ develops a strong and narrow peak near $E^{\prime} \simeq E$. To handle this we follow refs. $[39,53]$ and treat the electron energy loss due to IC in the Thomson limit as a continuous process by replacing

$$
-\Gamma_{e}(E) \bar{f}_{e}(E)+\int_{E}^{E_{N}} d E^{\prime} K_{e e}\left(E, E^{\prime}\right) \bar{f}_{e}\left(E^{\prime}\right) \rightarrow \frac{\partial}{\partial E}\left[\dot{E} \bar{f}_{e}(E)\right] .
$$

Here, $\dot{E}$ is the rate of energy loss from IC of a single electron in the photon background, given by [53]

$$
\frac{\dot{E}}{E}=-\frac{4}{3}\left[\frac{3 \zeta(4)}{\zeta(3)}\right]\left(\frac{E T}{m_{e}^{2}}\right) \sigma_{T} n_{\gamma}
$$

where $\sigma_{T}=(8 \pi / 3) \alpha^{2} / m_{e}^{2}$ is the Thomson cross section, $n_{\gamma}=\left[2 \zeta(3) / \pi^{2}\right] T^{3}$ is the thermal photon density, and $\zeta(z)$ is the Riemann zeta function. The approximation of eq. (2.11) is valid provided the fractional energy loss rate $\dot{E} / E$ is much smaller than the total scattering rate $\sigma_{T} n_{\gamma}$, which coincides with $y_{e} \ll 0.1$. In this limit, the two terms on the left-hand side of eq. (2.11) are much larger than their difference leading to a numerical instability in the original iterative approach.

When computing the electromagnetic spectra, we use the iterative method described above with eqs. (2.7), (2.8) until $y_{j}=E_{j} T / m_{e}^{2}<0.05$ is reached. For lower energy bins we keep eq. (2.7) for $\bar{f}_{\gamma}$ but apply the replacement of eq. (2.11) for $\bar{f}_{e}$, yielding the solution

$$
\bar{f}_{e}(E)=\left(\frac{E_{j}}{E}\right)^{2} \bar{f}_{e}\left(E_{j}\right)+\frac{1}{a_{T} E^{2}} \int_{E}^{E_{j}} d E^{\prime \prime} \mathcal{S}_{e}^{\prime}\left(E^{\prime \prime}\right)
$$


with

$$
\mathcal{S}_{e}^{\prime}\left(E^{\prime \prime}\right)=\xi_{\gamma} \frac{K_{e \gamma}\left(E^{\prime \prime}, E_{N}\right)}{\Gamma_{\gamma}\left(E_{N}\right)}+\int_{E^{\prime \prime}}^{E_{X}} d E^{\prime} K_{e \gamma}\left(E^{\prime \prime}, E^{\prime}\right) \bar{f}_{\gamma}\left(E^{\prime}\right)
$$

and

$$
a_{T}=\frac{\dot{E}}{E^{2}}=\frac{4 \pi^{2}}{45} \sigma_{T} \frac{T^{4}}{m_{e}^{2}} .
$$

Again, this can be evaluated iteratively, from high to low. While we use the specific value $y_{e}<0.05$ to match from one method to the other, we find nearly identical results from matching within the range $y_{e} \in[0.001,0.1]$.

\subsubsection{Monochromatic electron injection}

Monochromatic injection of electrons (and positrons) at energy $E_{X}$ can be treated nearly identically to monochromatic photon injection, with the only major change being in modifying the sources to

$$
\begin{aligned}
& S_{\gamma}(E)=S_{\gamma}^{\mathrm{FSR}}(E)+\sum_{b} \int_{E}^{E_{X}} d E^{\prime} K_{\gamma b}\left(E, E^{\prime}\right) \mathcal{N}_{b}\left(E^{\prime}\right), \\
& \mathcal{S}_{e}(E)=\xi_{e} R \delta\left(E-E_{X}\right)+\sum_{b} \int_{E}^{E_{X}} d E^{\prime} K_{e b}\left(E, E^{\prime}\right) \mathcal{N}_{b}\left(E^{\prime}\right),
\end{aligned}
$$

where $R$ is the decay (or annihilation) rate per unit volume, $\xi_{e}$ is the number of electrons plus positrons injected per decay, and $S_{\gamma}^{\mathrm{FSR}}(E)$ is a contribution to photons from finalstate radiation to be discussed in more detail below. For decays of the form $X \rightarrow e^{+}+e^{-}$ we have $R=n_{X}(t) / \tau_{X}$ and $\xi_{e}=2$, while for annihilation $X+\bar{X} \rightarrow e^{+}+e^{-}$the rate is $R=\langle\sigma v\rangle n_{X} n_{\bar{X}}$ and $\xi_{e}=2$.

Given these source terms, it natural to define the reduced spectra $\bar{f}_{a}(E)$ by

$$
\begin{aligned}
& \bar{f}_{\gamma}(E)=\frac{1}{R} \mathcal{N}_{\gamma}(E) \\
& \bar{f}_{e}(E)=\frac{1}{R} \mathcal{N}_{e}(E)-\frac{\xi_{e}}{\Gamma_{e}\left(E_{X}\right)} \delta\left(E-E_{X}\right)
\end{aligned}
$$

Applying this to eq. (2.2) with the sources of eqs. (2.16), (2.17), we obtain the relations

$$
\begin{aligned}
& \Gamma_{\gamma}(E) \bar{f}_{\gamma}(E)=\frac{S_{\gamma}^{\mathrm{FSR}}(E)}{R}+\xi_{e} \frac{K_{\gamma e}\left(E, E_{X}\right)}{\Gamma_{e}\left(E_{X}\right)}+\sum_{b} \int_{E}^{E_{X}} d E^{\prime} K_{\gamma b}\left(E, E^{\prime}\right) \bar{f}_{b}\left(E^{\prime}\right) \\
& \Gamma_{e}(E) \bar{f}_{e}(E)=\xi_{e} \frac{K_{e e}\left(E, E_{X}\right)}{\Gamma_{e}\left(E_{X}\right)}+\sum_{b} \int_{E}^{E_{X}} d E^{\prime} K_{e b}\left(E, E^{\prime}\right) \bar{f}_{b}\left(E^{\prime}\right)
\end{aligned}
$$

These equations can be solved using the same methods as described above for photon injection, including a matching in the Thomson limit using eq. (2.11).

A new feature that we include for electron injection is a contribution to the photon spectrum from final-state radiation (FSR) off the injected electron; $S_{\gamma}^{\mathrm{FSR}}(E)$ in eq. (2.16). 
For processes of the form $X \rightarrow e^{+}+e^{-}$or $X+\bar{X} \rightarrow e^{+}+e^{-}$with $X$ uncharged and $E_{X} \gg m_{e}$, this new source can be approximated by $[54,55]$

$$
S_{\gamma}^{\mathrm{FSR}}(E) \simeq \frac{R}{E_{X}} \frac{\alpha}{\pi} \frac{1+(1-x)^{2}}{x} \ln \left[\frac{4 E_{X}^{2}(1-x)}{m_{e}^{2}}\right] \Theta\left(1-\frac{m_{e}^{2}}{4 E_{X}^{2}}-x\right),
$$

where $x=E / E_{X}$. To be fully consistent, a corresponding subtraction should be made from the electron source. However, we find that this modifies the spectra by less than a percent. In contrast, we show below that the direct contribution to the photon spectrum from FSR can be the dominant one at higher energies when $E_{X} T / m_{e}^{2} \ll 1$, when the initial electrons scatter via IC with the photon background mainly in the Thomson regime.

\subsection{Review of the universal spectrum}

Many studies of the effects of electromagnetic energy injection on BBN approximate the photon spectrum with the so-called universal spectrum. This is a simple parametrization of the full calculations of the photon spectrum in refs. [39, 40]. It replaces the source terms (direct and cascade) in eq. (2.2) with a zeroeth generation spectrum $\mathcal{S}_{\gamma}(E) / R \rightarrow p_{\gamma}(E)$ based on the assumption that $4 \mathrm{P}$ and IC processes instantaneously reprocess the initial injected electromagnetic energy.

The standard parametrization used for the zeroeth generation spectrum is [4, 25, 39]

$$
p_{\gamma}\left(E_{\gamma}\right) \simeq \begin{cases}0 & ; E_{\gamma}>E_{c} \\ K_{0}\left(\frac{E_{\gamma}}{E_{m}}\right)^{-2.0} & ; E_{m}<E_{\gamma}<E_{c} \\ K_{0}\left(\frac{E_{\gamma}}{E_{m}}\right)^{-1.5} & ; E_{\gamma}<E_{m}\end{cases}
$$

where $E_{c} \simeq m_{e}^{2} / 22 T$ and $E_{m} \simeq m_{e}^{2} / 80 T$ are derived from ref. [40], and $K_{0}$ is a normalization constant. For monochromatic injection of $\xi$ photons, electrons, and positrons each with energy $E_{X}$, it is fixed by the requirement

$$
\xi E_{X}=\int_{0}^{E_{X}} d E E p_{\gamma}(E)
$$

implying $K_{0}=\xi E_{X} /\left[E_{m}^{2}\left(2+\ln \left(E_{c} / E_{m}\right)\right]\right.$ for $E_{X}>E_{c}$. An important feature of the spectrum is that it is proportional to the total injection energy (for either photons or electrons) provided $E_{X} \gg E_{c}$, up to an overall normalization by the total amount of energy injected.

Within the universal spectrum approximation, the final spectra are given by

$$
f_{\gamma}(E)=\frac{p_{\gamma}(E)}{\Gamma_{\gamma}(E)}, \quad f_{e}(E)=0
$$

where $f_{\gamma}(E)=\mathcal{N}_{\gamma}(E) / R$, and the relaxation rate $\Gamma_{\gamma}(E)$ accounts for the further reprocessing of the spectrum by slower processes like Compton scattering, pair creation on nuclei, and photon-photon scattering. ${ }^{2}$ These spectra have no residual delta-function parts since

\footnotetext{
${ }^{2}$ In practice, this $\Gamma_{\gamma}(E)$ is effectively equal to the full relaxation rate that also includes $4 \mathrm{P}$ scattering since this process is very strongly Boltzmann-suppressed for $E<E_{c}$.
} 

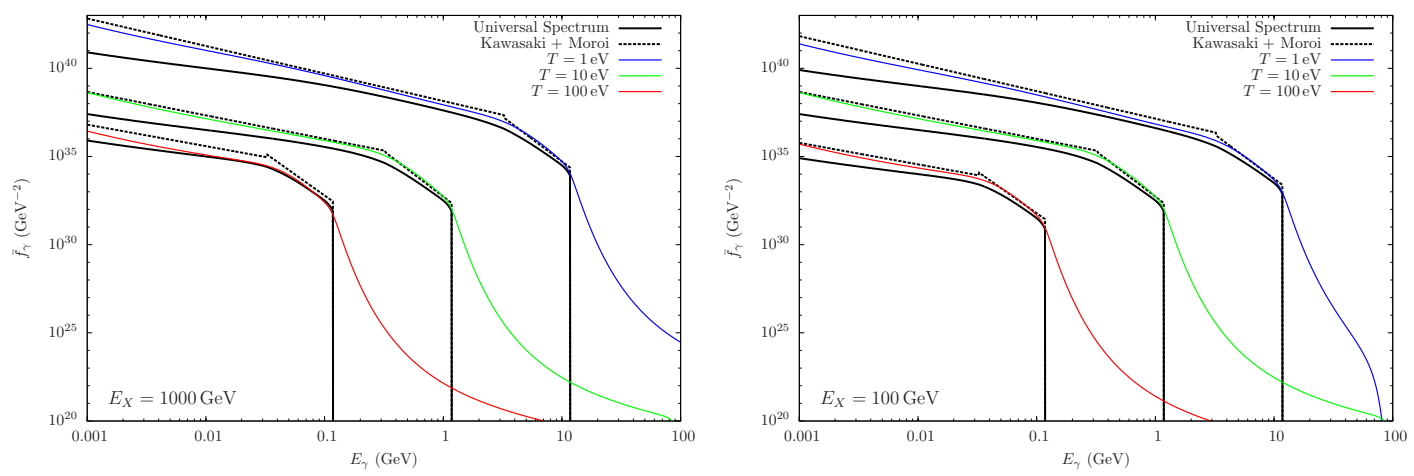

Figure 1. Photon spectrum $\bar{f}_{\gamma}(E)$ for single photon injection with energy $E_{X}=1000 \mathrm{GeV}$ (left) and $100, \mathrm{GeV}$ (right), for temperatures $T=1,10,100 \mathrm{eV}$. Also shown are the predictions of the universal spectrum (solid) and the parametrizations of Kawasaki and Moroi given in ref. [40].
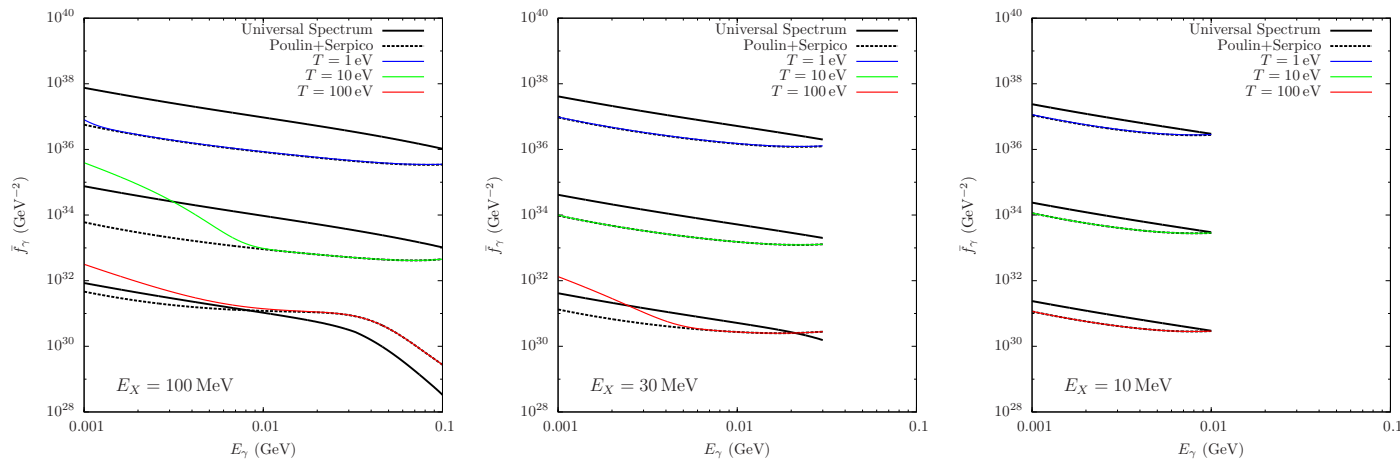

Figure 2. Photon spectrum $\bar{f}_{\gamma}(E)$ for photon injection with $E_{X}=100 \mathrm{MeV}$ (left), $E_{X}=$ $30 \mathrm{MeV}$ (middle), and $E_{X}=10 \mathrm{MeV}$ (right), with $T=1,10,100 \mathrm{eV}$. Also shown are the predictions of the universal spectrum (solid) and the low-energy prescription of ref. [52].

the initial injection is assumed to be fully reprocessed into the zeroeth-order spectrum by $4 \mathrm{P}$ and IC scatterings.

\subsection{Results for photon injection}

To validate our electromagnetic spectra, we compare our results to previous calculations and the universal spectrum at high injection energies. In figure 1 we show our photon spectra $\bar{f}_{\gamma}(E)$ for single photon injection with $E_{X}=1000 \mathrm{GeV}$ (left) and $100 \mathrm{GeV}$ (right) at temperatures $T=1,10,100 \mathrm{eV}$. Also shown in the figure are the predictions from the universal spectrum and parametrizations of the results of Kawasaki and Moroi listed in ref. [40]. In all cases here, $E_{X} \gg E_{c}$ and the universal spectrum is expected to be a good approximation. Our spectra agree well with the results of ref. [40] but are somewhat larger than the universal spectrum. We have also checked that our spectra scale proportionally to the total energy injected provided $E_{X} \gg E_{c}$. In all cases shown in the figure, the electron spectra are smaller than the photon spectra by orders of magnitude due to efficient IC scattering. Also visible is the strong suppression of the photon spectra for $E>E_{c}$ where the $4 \mathrm{P}$ process is active. 

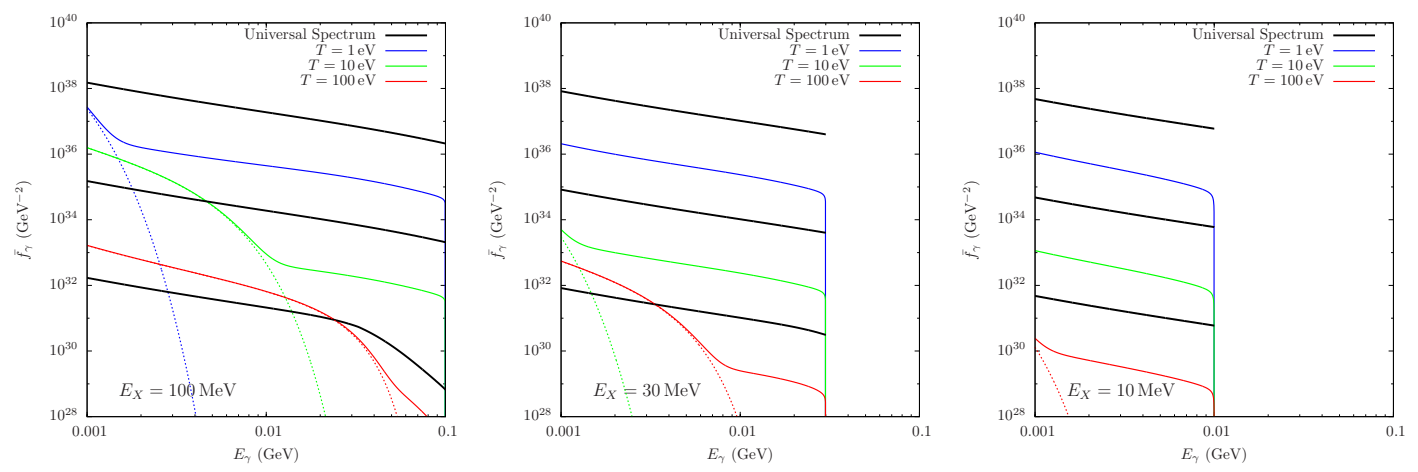

Figure 3. Photon spectrum $\bar{f}_{\gamma}(E)$ for electron plus positron $\left(e^{+} e^{-}\right)$injection with energies $E_{X}=$ $100 \mathrm{MeV}$ (left), $30 \mathrm{MeV}$ (middle), and $10 \mathrm{MeV}$ (right), with $T=1,10,100 \mathrm{eV}$. The solid lines show the full spectrum, while the dashed lines show the result when FSR is not taken into account. Also shown is the universal spectrum for the same total injected energy.

In contrast to electromagnetic injection at high energies with $E_{X} \gg E_{c}$, injection at lower energies with $E_{X} \lesssim E_{c}$ has received much less attention. In figure 2 we show our computed photon spectra for single photon injection with $E_{X}=100 \mathrm{MeV}$ (left), $E_{X}=$ $30 \mathrm{MeV}$ (middle), and $E_{X}=10 \mathrm{MeV}$ (right) for $T=1,10,100 \mathrm{eV}$. Also shown are the predictions of the universal spectrum (normalized according to eq. (2.24)) and the prescription by Poulin and Serpico of ref. [52]. Since $E_{X}<E_{c}$, the assumptions that go into the universal spectrum are not met and it is not expected to be accurate in this regime, as first pointed out in ref. [52]. Our spectra agree fairly well with the results of ref. [52], which only kept the photon part of the spectrum. Some deviations are seen at lower energies where photon regeneration by IC becomes significant. Note as well that the full cascade also contains a moderately damped delta-function part that is not shown here (and was explicitly removed in our definition of $\bar{f}_{\gamma}$ in eq. (2.5)).

\subsection{Results for electron injection}

For electron and positron $\left(e^{+} e^{-}\right)$injection with energies $E_{X} \gg E_{c}$, we find the same photon (and electron) spectra as from photon injection with an equal total input energy, and thus our results agree reasonably well with ref. [40] and the universal spectrum in this limit. However, for $E_{X} \lesssim E_{c}$ we find very significant variations from the universal spectrum as well as from pure photon injection. Photon spectra $\bar{f}_{\gamma}$ resulting from $e^{+} e^{-}$injection are shown in figure 3 for input energies $E_{X}=100 \mathrm{MeV}$ (left), $E_{X}=30 \mathrm{MeV}$ (middle), and $E_{X}=10 \mathrm{MeV}$ (right) and temperatures $T=1,10,100 \mathrm{eV}$. The solid lines show the full spectra, while the dashed lines show the corresponding result when FSR off the initial decay electrons is not taken into account. Also shown is the universal spectrum for the same total energy injection (normalized according to eq. (2.24)). Let us also mention that the photon spectra do not have a delta function component for electron or positron injection.

The strong suppression of the photon spectrum from electron injection at lower energies in the absence of FSR was pointed out in ref. [42]. As argued there, this suppression can be understood in terms of the behavior of IC scattering at low energy, which is the main 


\begin{tabular}{|ccc|}
\hline Process & Threshold $(\mathrm{MeV})$ & Peak value (mb) \\
\hline $\mathrm{D}+\gamma \rightarrow p+n[56]$ & 2.220 & 2.47 \\
${ }^{3} \mathrm{He}+\gamma \rightarrow p+p+n[57]$ & 2.486 & 1.02 \\
${ }^{3} \mathrm{He}+\gamma \rightarrow \mathrm{D}+p[57]$ & 5.490 & 1.18 \\
$\mathrm{~T}+\gamma \rightarrow n+\mathrm{D}[58,59]$ & 6.260 & 0.818 \\
$\mathrm{~T}+\gamma \rightarrow n+n+p[59]$ & 8.480 & 0.878 \\
${ }^{4} \mathrm{He}+\gamma \rightarrow \mathrm{T}+p[60]$ & 19.81 & 1.31 \\
${ }^{4} \mathrm{He}+\gamma \rightarrow{ }^{3} \mathrm{He}+n[61,62]$ & 20.58 & 1.28 \\
${ }^{4} \mathrm{He}+\gamma \rightarrow \mathrm{D}+\mathrm{D}[25]$ & 23.85 & 0.0051 \\
${ }^{4} \mathrm{He}+\gamma \rightarrow n+p+\mathrm{D}[60]$ & 26.07 & 0.182 \\
\hline
\end{tabular}

Table 1. Processes included in our calculation of photodissociation effects from electromagnetic injections, as well as their threshold energies and peak cross sections.

mechanism for electrons to transfer energy to photons in this context. For smaller $E_{X}$ and $T$, the dimensionless combination $y_{e}=E_{e} T / m_{e}^{2} \ll 1$ is small, and IC scattering lies in the Thomson regime where each collision only slightly reduces the initial electron energy. Correspondingly, the maximal scattered photon energy $E_{\gamma}^{\prime}$ in the Thomson limit is $E_{\gamma}^{\prime} \leq 4\left(E_{e} / m_{e}\right) E_{\gamma}$, where $E_{\gamma}$ is the energy of the initial photon. Since the initial photon comes from the CMB, $E_{\gamma} \sim T$ is expected so that

$$
\begin{aligned}
E_{\gamma}^{\prime} & \lesssim 4\left(E_{e} / m_{e}\right) T \\
& \sim 15 \mathrm{MeV}\left(\frac{E_{e}}{100 \mathrm{MeV}}\right)^{2}\left(\frac{T}{100 \mathrm{eV}}\right) .
\end{aligned}
$$

Higher scattered photon energies are possible, but they come at the cost of an exponential Boltzmann suppression.

In this regime, FSR from the injected electrons and positrons can be the dominant contribution to the photon spectrum, as illustrated in figure 3. Relative to the rest of the cascade, the distribution of photons from FSR is hard, falling off roughly as $1 / E$ instead of as $1 / E^{2}$. Despite the suppression of FSR by $(\alpha / \pi) \times \log$ (with $\log \sim$ few), it can easily overcome the exponential suppression of IC for photon energies above the bound of eq. (2.26). We show below that this has a very important implication for the effects of lower-energy electron injection on the primordial light element abundances. Note, however, that FSR has only a very minor effect on the spectra for photon injection or when $E_{X} \gg E_{c}$.

\section{Effects of electromagnetic injection on $\mathrm{BBN}$}

Having computed the electromagnetic cascades from lower-energy injection, we turn next to investigate the effects of such injection on the primordial element abundances from BBN.

\subsection{Photodissociation of light elements}

Photodissociation of light element begins when the temperature of the cosmological plasma falls low enough for MeV photons to populate the electromagnetic cascade. From eq. (1.1), 
this does not begin until temperatures fall below about $10 \mathrm{keV}$ (corresponding to $t \sim 10^{4} \mathrm{~s}$ ). By this time element creation by BBN has effectively turned off, and thus we can compute the effects of photodissociation as a post-processing of the outputs of standard BBN [3, 25].

The effects of photodissociation on the light element abundances can be described by a set of coupled Boltzmann equations of the form

$$
\frac{d Y_{A}}{d t}=\sum_{i} Y_{i} \int_{0}^{\infty} d E_{\gamma} \mathcal{N}_{\gamma}\left(E_{\gamma}\right) \sigma_{\gamma+i \rightarrow A}\left(E_{\gamma}\right)-Y_{A} \sum_{f} \int_{0}^{\infty} d E_{\gamma} \mathcal{N}_{\gamma}\left(E_{\gamma}\right) \sigma_{\gamma+A \rightarrow f}\left(E_{\gamma}\right),
$$

where $\mathcal{N}_{\gamma}\left(E_{\gamma}\right)$ are the photon spectra calculated above, $A$ and the sums run over the relevant isotopes, and $Y_{A}$ are number densities normalized to the entropy density,

$$
Y_{A}=\frac{n_{A}}{s} \text {. }
$$

Note that we do not include reactions initiated by electrons because the electron spectra are always strongly suppressed by IC scattering.

In our analysis we include the nuclear species hydrogen $(H)$, deuterium $\left(\mathrm{D}={ }^{2} \mathrm{H}\right)$, tritium $\left(\mathrm{T}={ }^{3} \mathrm{H}\right)$, helium-3 $\left({ }^{3} \mathrm{He}\right)$, and helium $\left(\mathrm{He}={ }^{4} \mathrm{He}\right)$. Heavier species including lithium isotopes could also be included, but these have much smaller abundances and they would not alter the results for the lighter elements we consider. The nuclear cross sections included in our study are listed in table 3.1, for which we use the simple parametrizations of ref. [25]. All these cross sections have the same general shape as a function of energy, with a sharp rise at the threshold up to a peak followed by a smooth fall off. We list the threshold energies and peak values of the cross sections in the table to give an intuitive picture of their relevant strengths and ranges of importance. Of the nine cross sections listed, it is helpful to group them into processes that destroy helium and create deuterium and helium-3 with thresholds above $20 \mathrm{MeV}$, and processes that destroy the lighter isotopes with significantly lower thresholds.

It is straightforward to solve the evolution equations of eq. (3.1) numerically following the standard convention of converting the dependent variable from time to redshift. For standard BBN values of the primordial abundances, we use the predictions of PArthENoPE [63, 64]:

$$
Y_{p}=0.247, \quad \frac{n_{\mathrm{D}}}{n_{\mathrm{H}}}=2.45 \times 10^{-5}, \quad \frac{n_{3} \mathrm{He}}{n_{\mathrm{H}}}=0.998 \times 10^{-5} .
$$

In the analysis to follow, we compare the computed output densities to the following observed values, quoted with effective $1 \sigma$ uncertainties into which we have combined theoretical and experimental uncertainties in quadrature:

$$
\begin{aligned}
Y_{p} & =0.245 \pm 0.004 & & (\text { Ref. [65]) } \\
\frac{n_{\mathrm{D}}}{n_{\mathrm{H}}} & =(2.53 \pm 0.05) \times 10^{-5} & & (\text { Ref. [66]) } \\
\frac{n_{3} \mathrm{He}}{n_{\mathrm{H}}} & =(1.0 \pm 0.5) \times 10^{-5} & & \text { (Ref. [67]) }
\end{aligned}
$$

For the helium mass fraction $Y_{p}$, the value we use is consistent with ref. [68] and previous determinations but significantly lower than the determination of ref. [69]. The quoted 

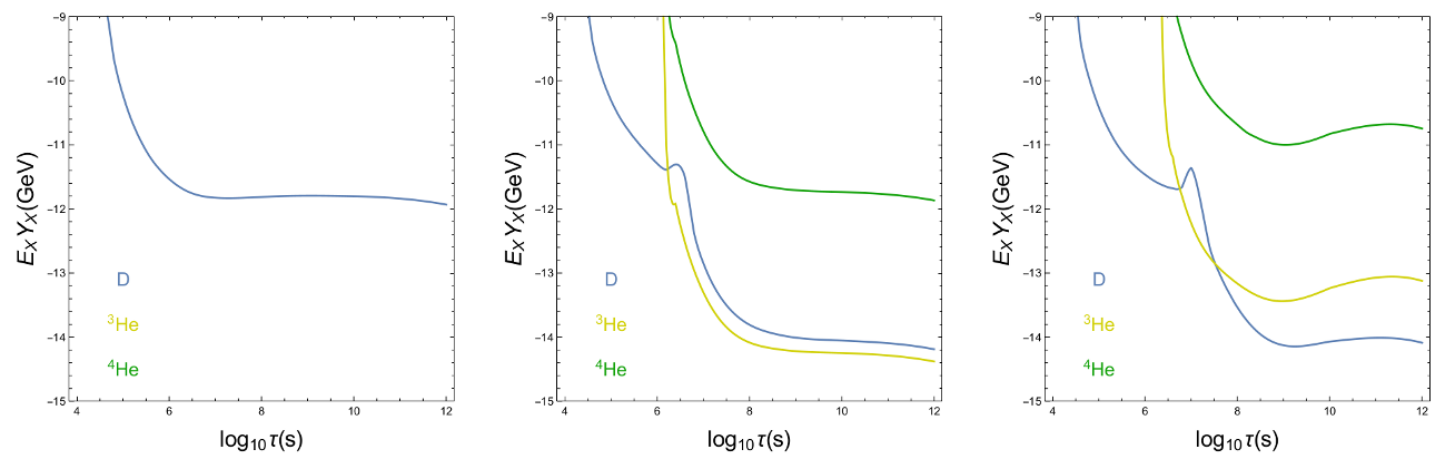

Figure 4. Limits on $E_{X} Y_{X}$ from $\mathrm{BBN}$ on the monochromatic photon decay of species $X$ as a function of the lifetime $\tau_{X}$ for photon injection energies $E_{X}=10 \mathrm{MeV}$ (left), $30 \mathrm{MeV}$ (middle), and $100 \mathrm{MeV}$ (right). Bounds are given for the effects on the nuclear species D, ${ }^{3} \mathrm{He}$, and ${ }^{4} \mathrm{He}$.

uncertainty on the ratio $n_{\mathrm{D}} / n_{\mathrm{H}}$ is dominated by a theory uncertainty on the rate of photon capture on deuterium from ref. [70]. For $n_{3} \mathrm{He}_{\mathrm{e}} / n_{\mathrm{H}}$, we use the determination of $\left(n_{\mathrm{D}}+\right.$ $\left.n_{3} \mathrm{He}\right) / n_{\mathrm{H}}$ of ref. [67] together with the value of $n_{\mathrm{D}} / n_{\mathrm{H}}$ from ref. [66]; the resulting upper bound (with uncertainties) is similar to but slightly stronger than what is used in ref. [30]. The uncertainties quoted here are generous, and in the analysis to follow we implement exclusions at the $2 \sigma$ level.

\subsection{BBN constraints on photon injection}

Following the methods described above and the electromagnetic cascades computed previously, we derive BBN bounds on monochromatic photon injection from late decays with lifetime $\tau_{X}$ and initial injection energy $E_{X}$. In figure 4 we show the resulting limits on the combination $E_{X} Y_{X}$, where $Y_{X}$ is the predecay yield of the decaying species $X$ (assumed to produce one photon per decay) for injection energies $E_{X}=10,30,100 \mathrm{MeV}$. The bounds coming from $\mathrm{D},{ }^{3} \mathrm{He}$, and ${ }^{4} \mathrm{He}$ are shown individually, and correspond to $2 \sigma$ exclusions. Early on, when $E_{c}$ is small, the dominant effect is destruction of $\mathrm{D}$ since it has the lowest photodissociation threshold. Later on, as $E_{c}$ increases, it becomes possible to create excess $\mathrm{D}$ and ${ }^{3} \mathrm{He}$ through the destruction of ${ }^{4} \mathrm{He}$ provided the injection energy is larger than the ${ }^{4} \mathrm{He}$ threshold of about $20 \mathrm{MeV}$. Destruction of $\mathrm{D}$ is the dominant effect at all times for $E_{X}$ below the helium threshold, as can be seen in the leftmost panel of figure 4 .

In figure 5 we show maximal values of $E_{X} Y_{X}$ from monochromatic photon injection at energy $E_{X}$ from the decay of species $X$ as a function of $\tau_{X}$ and $E_{X}$. The combined exclusion is based on the union of $2 \sigma$ exclusions of the individual species. Clear features are visible in this figure at $\tau_{X} \simeq 10^{6} \mathrm{~s}$ and $E_{X} \simeq 20 \mathrm{MeV}$. These coincide with the structure of the exclusions shown in figure 4 , with both corresponding to where the photodissociation of ${ }^{4} \mathrm{He}$ turns off, either because $E_{c}$ or $E_{X}$ is too small.

\subsection{BBN constraints on electron injection}

In figure 6 we show the limits for $e+e^{-}$injection from the decay of a species $X$ with lifetime $\tau_{X}$ on $E_{X} Y_{X}$, where $Y_{X}$ is the predecay yield of the decaying species $X$ (assumed to produce 


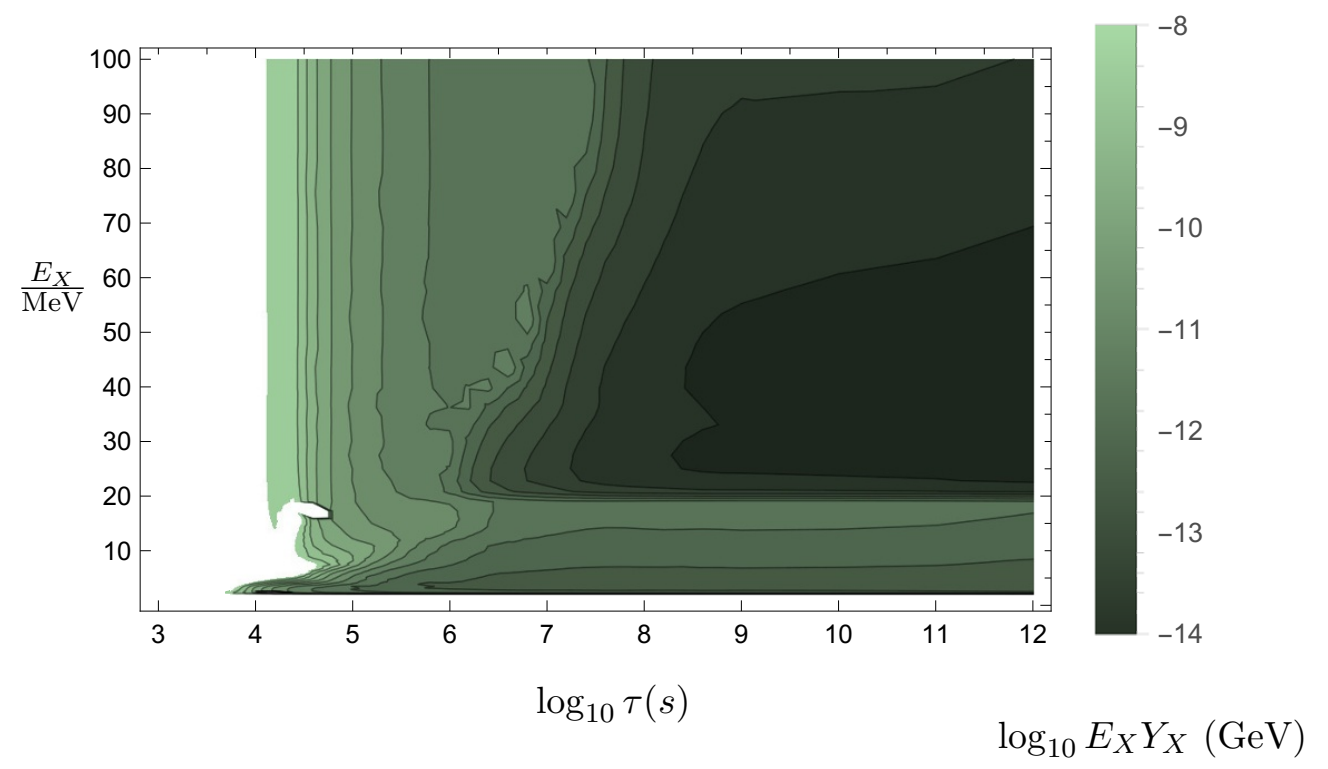

Figure 5. Combined limits on $E_{X} Y_{X}$ as a function of $\tau_{X}$ and $E_{X}$ for the decay of a species $X$ with lifetime $\tau_{X}$ injecting a single photon with energy $E_{X}$.
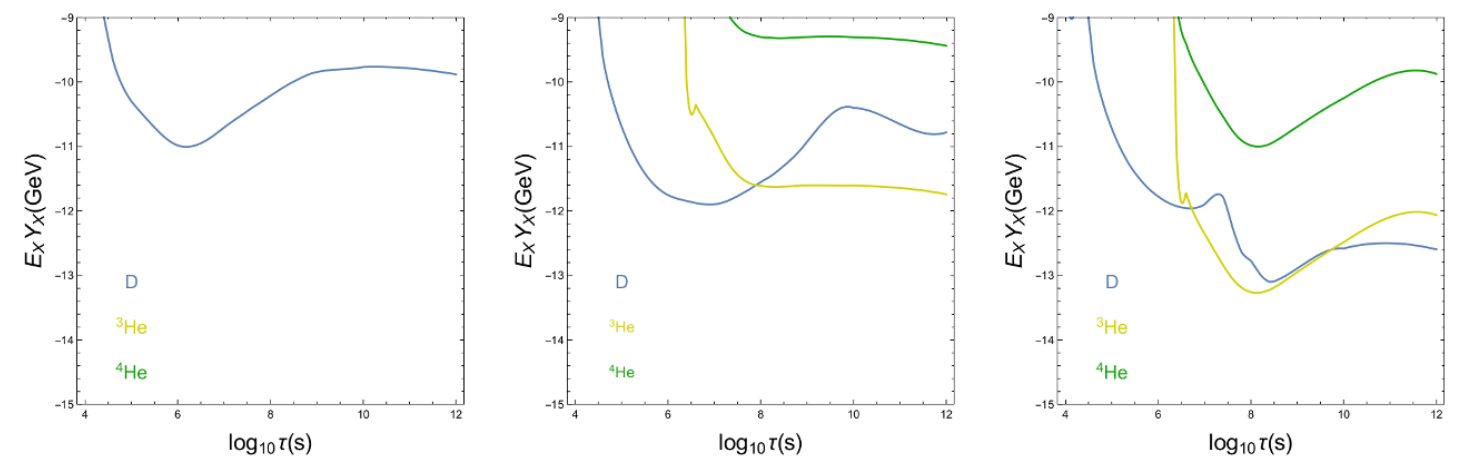

Figure 6. Limits on $E_{X} Y_{X}$ from BBN on the monochromatic $e^{+} e^{-}$decay of species $X$ as a function of the lifetime $\tau_{X}$ for individual electron injection energies $E_{X}=10 \mathrm{MeV}$ (left), $30 \mathrm{MeV}$ (middle), and $100 \mathrm{MeV}$ (right). Bounds are given for the effects on the nuclear species $\mathrm{D},{ }^{3} \mathrm{He}$, and ${ }^{4} \mathrm{He}$, and contributions to the electromagnetic cascades from FSR are included.

one $e^{+} e^{-}$pair per decay) for injection energies for each electron of $E_{X}=10,30,100 \mathrm{MeV}$ (from left to right). The bounds coming from $\mathrm{D},{ }^{3} \mathrm{He}$, and ${ }^{4} \mathrm{He}$ are shown individually, and correspond to $2 \sigma$ exclusions. The electromagnetic spectra used in this calculation include FSR from the injected $e^{+} e^{-}$pair. The resulting bounds are somewhat weaker than for photon injection and follow a similar pattern, and remain quite strong even down to $E_{X}=10 \mathrm{MeV}$. For comparison, we show the corresponding results when FSR effects are not included in figure 7. As expected, the exclusions are significantly weaker, particularly for larger $\tau_{X}$ and lower $E_{X}$ where the relevant IC scattering is deep in the Thomson regime.

In figure 8 we show maximal values of $E_{X} Y_{X}$ from monochromatic $e^{+} e^{-}$injection at energy $E_{X}$ from the decay of species $X$ as a function of $\tau_{X}$ and $E_{X}$, with FSR effects 

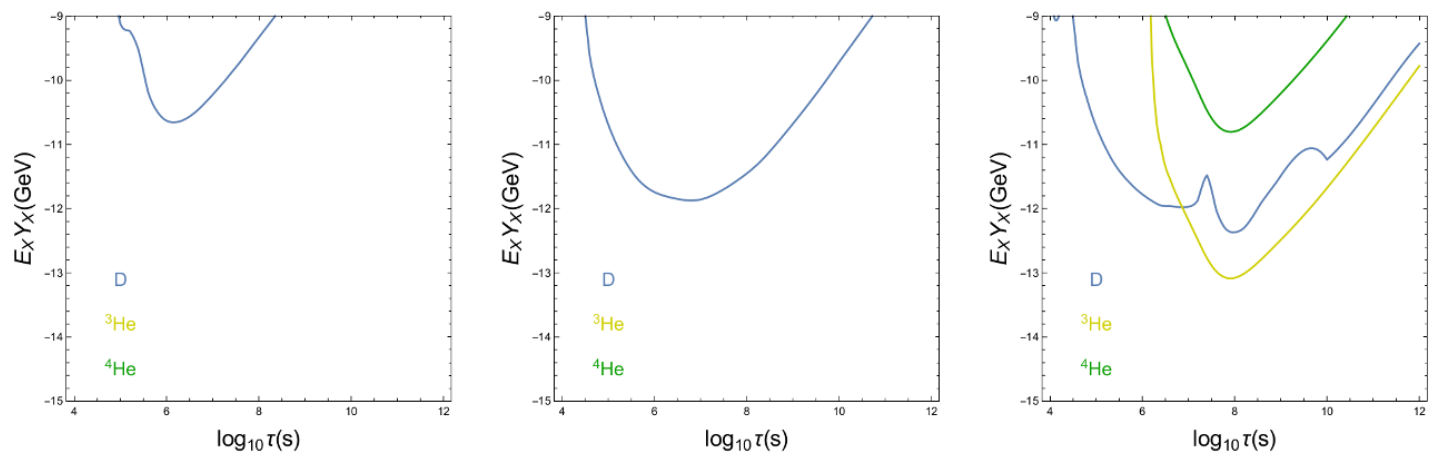

Figure 7. Same as figure 6 but without FSR effects.

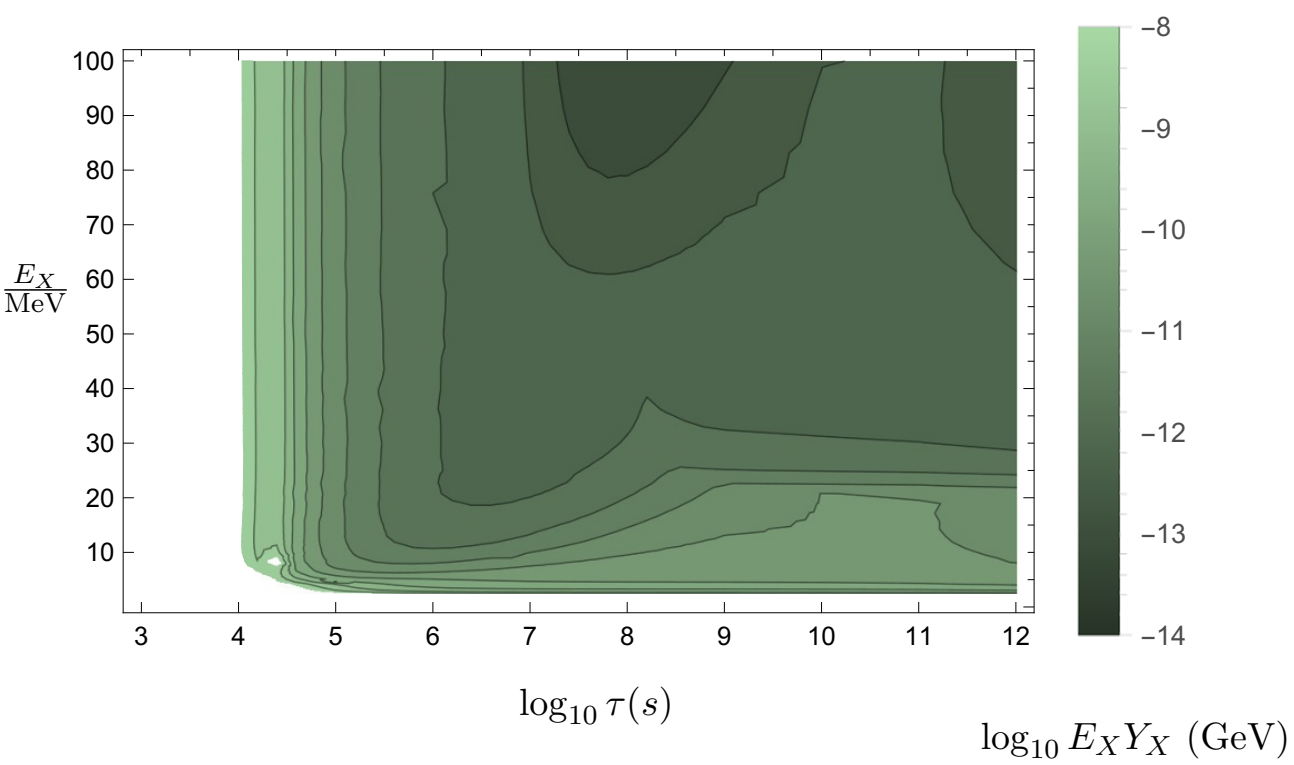

Figure 8. Combined limits on $E_{X} Y_{X}$ as a function of $\tau_{X}$ and $E_{X}$ for the decay of a species $X$ with lifetime $\tau_{X}$ injecting an electron-positron pair each with energy $E_{X}$, with FSR effects included.

included in the electromagnetic cascade. The combined exclusion is based on the union of $2 \sigma$ exclusions of the individual species. Again, the exclusions become weaker for $\tau_{X} \lesssim 10^{6} \mathrm{~s}$ or $E_{X} \lesssim 20 \mathrm{MeV}$ where the photodissociation of ${ }^{4} \mathrm{He}$ turns off. The bounds on $e^{+} e^{-}$ injection are also typically weaker than for photon injection, but not drastically so when FSR is taken into account.

\section{Other constraints on electromagnetic decays}

In addition to modifying the primordial light element abundances, energy injection in the early universe can produce other deviations from the standard cosmology. Electromagnetic decays near or after recombination at $t_{\mathrm{rec}} \simeq 1.2 \times 10^{13} \mathrm{~s}$ can modify the temperature and polarization power spectra of the CMB [71-74]. Since current CMB observations are found to constrain such decays much more strongly than $\mathrm{BBN}[75,76]$, we focus here on decays 
prior to recombination. The best limits in this case, aside from BBN, come from entropy injection and modifications to the CMB frequency spectrum. In this section we estimate these other limits on late energy injection and compare them to our results for BBN.

Entropy injection after the start of BBN leads to a lower measured baryon density today relative to the value deduced from BBN. This was studied in ref. [77] with the result

$$
\frac{\Delta s}{s} \simeq 7.8 \times 10^{-5}\left(\frac{\Delta E Y_{X}}{10^{-10} \mathrm{GeV}}\right)\left(\frac{\tau}{10^{6} \mathrm{~s}}\right)^{1 / 2},
$$

where $\Delta E$ is the total electromagnetic energy injected per decay and $\tau_{X} \gtrsim 1 \mathrm{~s}$. A related constraint can be derived for variations in the effective number of neutrinos $N_{\text {eff }}$ from photon heating after neutrino decoupling [37, 38, 50, 78].

Late decays releasing electromagnetic energy can also distort the frequency spectrum of the CMB [79, 80], which is observed to be a nearly-perfect blackbody [81]. The effect depends on the decay time $\tau_{X}$ relative to the times $\tau_{d C} \simeq 6.1 \times 10^{6} \mathrm{~s}$ when double-Compton scattering freezes out and $\tau_{C} \simeq 8.8 \times 10^{9} \mathrm{~s}$ when Compton scattering turns off $[79,80]$. Decays with $\tau_{d C}<\tau_{X}<\tau_{C}$ yield products that thermalize through Compton scattering and generate an effective photon chemical potential $\mu$ given by [79, 80, 82]

$$
\mu \simeq 5.6 \times 10^{-4}\left(\frac{\Delta E Y_{X}}{10^{-10} \mathrm{GeV}}\right)\left(\frac{\tau}{10^{6} \mathrm{~s}}\right)^{1 / 2} e^{-\left(\tau_{d C} / \tau\right)^{5 / 4}} .
$$

For $\tau_{X}>\tau_{C}$, electromagnetic injection produces a distortion that can be described by the Compton parameter $y=\Delta \rho_{\gamma} / 4 \rho_{\gamma}$, with the approximate result $[79,80,82]$

$$
y \simeq 5.7 \times 10^{-5}\left(\frac{\Delta E Y_{X}}{10^{-10} \mathrm{GeV}}\right)\left(\frac{\tau}{10^{6} \mathrm{~s}}\right)^{1 / 2} \mathcal{C}(\tau),
$$

where $\mathcal{C}(\tau)=1$ for $\tau<t_{\text {eq }}$ and $\mathcal{C}(\tau) \simeq\left(\tau / t_{\text {eq }}\right)^{1 / 6}$ for $\tau>t_{\text {eq }}$. The current limits on $\mu$ and $y$ are [81]

$$
\mu<9 \times 10^{-5}, \quad|y|<1.5 \times 10^{-5},
$$

while the proposed PIXIE satellite is to have sensitivity to constrain [83]

$$
\mu<1 \times 10^{-8}, \quad|y|<2 \times 10^{-9} .
$$

In the left and right panels of figure 9 we show the limits from entropy injection and $\mathrm{CMB}$ spectral distortions. The solid red line shows $\Delta s / s=0.1$, and demanding variations below this is a conservative requirement relative to those imposed in refs. [50, 52]. For CMB spectral distortions we show bounds on the $\mu$ and $y$ parameters in blue based on the approximate estimates above based on measurements by COBE/FIRAS (solid) and the projected sensitivity of PIXIE (dotted). For comparison, we show in green the limits derived above for monochromatic photon injection (left) and monochromatic $e^{+} e^{-}$ injection (right). In both panels, the dotted, dashed, and solid lines correspond to injection with $E_{X}=10,30,100 \mathrm{MeV}$. Even for low injection energies, BBN constraints currently dominate for $\tau \gtrsim 10^{4} \mathrm{~s}$ until being replaced by bounds from either CMB frequency or power spectrum variations. Even with the vast improvement expected from PIXIE, BBN will continue to provide the strongest limit on electromagnetic decays in the early universe with lifetimes $10^{4} \mathrm{~s} \lesssim \tau_{X} \lesssim 10^{6} \mathrm{~s}$ and energy injections above a few $\mathrm{MeV}$. 

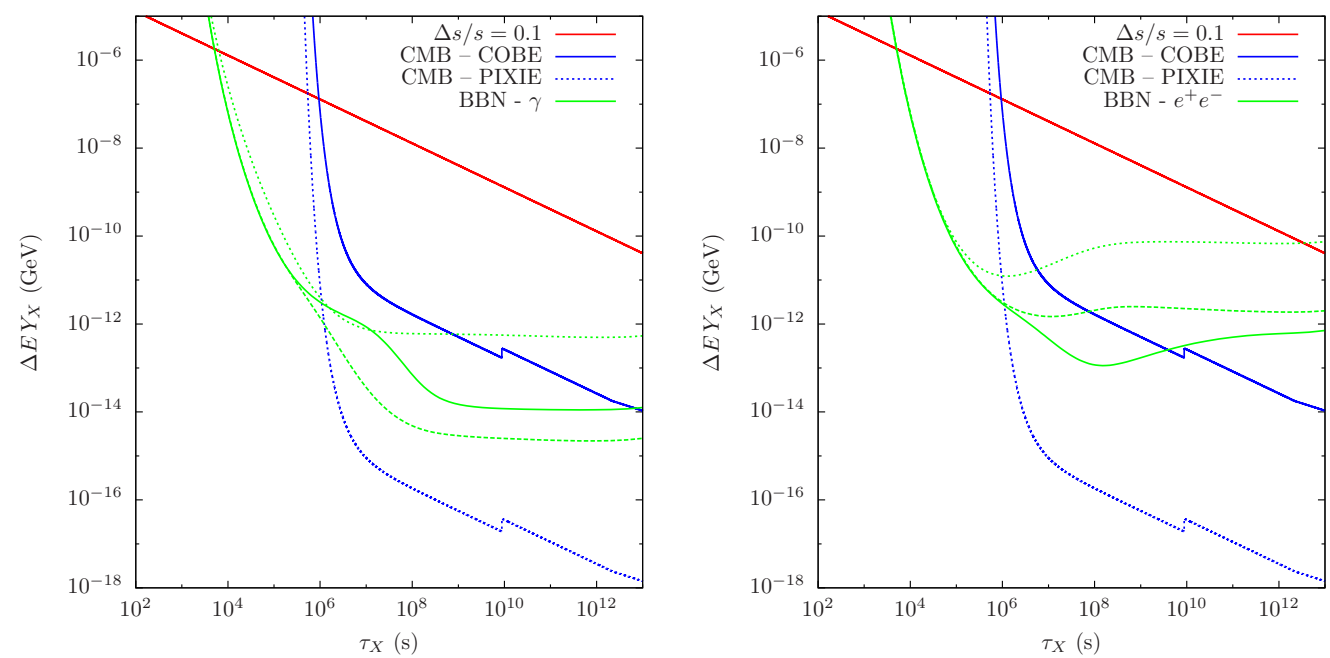

Figure 9. Other bounds on electromagnetic decays in the early universe as a function of the lifetime $\tau_{X}$ and the total electromagnetic injection $\Delta E Y_{X}$ relative to limits derived from BBN. In both panels, the red line shows $\Delta s / s=0.1$, while the solid (dotted) blue lines show the current and projected CMB frequency bounds from COBE/FIRAS (PIXIE). The left panel also indicates the limits derived from $\mathrm{BBN}$ for photon injection with energy $E_{X}=10,30,100 \mathrm{MeV}$ with green dotted, dashed, and solid lines. The right panel shows the corresponding BBN bounds from monochromatic $e^{+} e^{-}$injection.

\section{Conclusions}

In this paper we have investigated the electromagnetic cascades induced by electromagnetic energy injection in the range $E_{X}=1-100 \mathrm{MeV}$ and we have studied its effects on the light elements abundances created during BBN. As in ref. [52], we find significant deviations from the universal photon spectrum for monochromatic initial photon injection with energy $E_{X} \lesssim E_{c}=m_{e}^{2} / 22 T$. We also study how this impacts BBN. Our study also expands on previous work by computing the full electromagnetic cascade including electrons. For either photon or electron injection, we find that BBN provides the strongest constraint on late-decaying particles with lifetimes between $10^{4} \mathrm{~s} \lesssim \tau \lesssim 10^{13}$ s for electromagnetic energies nearly all the way down to the photodissociation threshold of deuterium near $E_{t h} \simeq 2.22, \mathrm{MeV}$.

Photon and electron injection produce very similar electromagnetic cascades for $E_{X} \gg$ $E_{c}$ but differ in important ways for $E_{X} \lesssim E_{c}$. Initial hard photons induce a smooth population of lower-energy photons through Compton and photon-photon scattering. In contrast, electrons injected with $E_{X} \lesssim m_{e}^{2} / 10 T$ interact mainly through inverse Compton (IC) scattering off the CMB, which lies in the Thomson regime at such energies. The upscattered photons from Thomson scattering have much lower energy than the initial electron, and can easily fall below the $\mathrm{MeV}$ scales needed to induce photodissociation. However, in this regime we find that photons radiated off the initial hard electrons can populate and dominate the induced photon spectrum up to near the initial electron energy. To our knowledge, the contribution of FSR to the photon spectrum has not been considered before in this 


\begin{tabular}{|c|c|c|}
\hline & Processes & Equations \\
\hline$\Gamma_{\gamma}$ & $4 \mathrm{P}, \mathrm{PP}, \mathrm{PCN}, \mathrm{CS}$ & $(\mathrm{A} .1),(\mathrm{A} .18),(\mathrm{A} .20),(\mathrm{A} .28)$ \\
$\Gamma_{e}$ & IC & (A.9) \\
$K_{\gamma \gamma}$ & $\mathrm{PP}, \mathrm{CS}$ & $(\mathrm{A} .19),(\mathrm{A} .30)$ \\
$K_{\gamma e}$ & IC & $(\mathrm{A} .17)$ \\
$K_{e \gamma}$ & $4 \mathrm{P}, \mathrm{PCN}, \mathrm{CS}$ & (A.5), $(\mathrm{A} .24),(\mathrm{A} .32)$ \\
$K_{e e}$ & IC & $(\mathrm{A} .15)$ \\
\hline
\end{tabular}

Table 2. Summary of the contributions to relaxation rates and energy transfer functions.

context since its effects are very small at the higher initial injection energies that have been investigated in the greatest detail.

While this work has concentrated on decays, our results for electromagnetic cascades are also applicable to annihilation in the early universe. Our results could also be used to investigate potential solutions to the apparent anomalies in the lithium abundances, which was studied in refs. $[50,84]$ using the universal spectrum.

Note added: as this work was nearing completion, ref. [85] appeared investigating many of the same topics including the development of the electromagnetic cascades from sub$100 \mathrm{MeV}$ decays. In the cases that are directly comparable, our results appear to be in substantial agreement.

\section{Acknowledgments}

We thank Sonia Bacca, Nikita Blinov, David Curtin, Barry Davids, David McKeen, Maxim Pospelov, and Adam Ritz for helpful discussions. DEM and GW thank the Aspen Center for Physics, which is supported by National Science Foundation grant PHY-1607611, for their hospitality while this work was being completed. This work is supported by the Natural Sciences and Engineering Research Council of Canada (NSERC), with DEM supported in part by Discovery Grants and LF by a CGS D scholarship. TRIUMF receives federal funding via a contribution agreement with the National Research Council of Canada.

\section{A Relaxation and transfer rates}

In this appendix we collect explicit expressions for the relevant relaxation rates and transfer functions. The processes included for each are listed in table 2 together with links to the corresponding equations.

\section{A.1 Rates for Photon Photon Pair Production (4P)}

The $4 \mathrm{P}$ process plays a key role in determining the electromagnetic cascade for $E>E_{c}$ and contributes to $\Gamma_{\gamma}$ and $K_{e \gamma}$. 


\section{A.1.1 4P photon relaxation}

The relevant expression is given in ref. [40]

$$
\Delta \Gamma_{\gamma}\left(E_{\gamma}\right)=\left.\frac{1}{8} \frac{1}{E_{\gamma}^{2}} \int_{m_{e}^{2} / E_{\gamma}}^{\infty} d \bar{E} \frac{1}{\pi^{2}}\left(e^{\bar{E} / T}-1\right)^{-1} \int_{4 m_{e}^{2}}^{4 E_{\gamma} \bar{E}} d s s \sigma_{4 P}(\beta)\right|_{\beta=\sqrt{1-4 m_{e}^{2} / s}}
$$

where

$$
\sigma_{4 P}(\beta)=\frac{\pi}{2} \frac{\alpha^{2}}{m_{e}^{2}}\left(1-\beta^{2}\right)\left[\left(3-\beta^{4}\right) \ln \left(\frac{1+\beta}{1-\beta}\right)-2 \beta\left(2-\beta^{2}\right)\right] .
$$

This expression can be written in the form

$$
\Delta \Gamma_{\gamma}\left(E_{\gamma}\right)=\frac{2}{\pi} \alpha^{2}\left(\frac{T}{m_{e}}\right)^{3} m_{e} \frac{1}{y_{\gamma}^{2}} \mathcal{G}\left(y_{\gamma}\right),
$$

where $y_{\gamma}=E_{\gamma} T / m_{e}^{2}$ and a function $\mathcal{G}\left(y_{\gamma}\right)$. A good numerical fit to this function is

$$
\frac{1}{y_{\gamma}^{2}} \mathcal{G}\left(y_{\gamma}\right) \simeq e^{-1 / y_{\gamma}}\left[(1.2) y_{\gamma}^{-0.425} e^{-\left(0.1 y_{\gamma}\right)^{2}}+(3.3) y_{\gamma}^{-0.85} e^{-\left(9 / y_{\gamma}\right)^{2}}\right]
$$

which is valid to better than about $10 \%$ for $y_{\gamma} \in\left[10^{-5}, 10^{2}\right]$. A similar approximate expression is given in ref. [39].

\section{A.1.2 4P transfer to electrons}

The $4 \mathrm{P}$ process also produces high energy electrons and positrons, contributing to the transfer function $K_{e \gamma}$ by $[40,86]:^{3}$

$$
\Delta K_{e \gamma}\left(E_{e}, E_{\gamma}\right)=\frac{\alpha^{2} m_{e}^{2}}{2 \pi} \frac{1}{E_{\gamma}^{3}} \int_{0}^{\infty} d \bar{E}_{\gamma}\left(e^{\bar{E}_{\gamma} / T}-1\right)^{-1} G\left(E_{e}, E_{\gamma}, \bar{E}_{\gamma}\right) \Theta\left(\left\{E_{i} ; T\right\}\right),
$$

where

$$
G\left(E_{e}, E_{\gamma}, \bar{E}_{\gamma}\right)=4 A \ln \left(\frac{4 B}{A}\right)-(1-1 / B) A^{2}+2 A(2 B-1)-8 B
$$

with

$$
A=\frac{\left(E_{\gamma}+\bar{E}_{\gamma}\right)^{2}}{E_{e}\left(E_{\gamma}+\bar{E}_{\gamma}-E_{e}\right)}, \quad B=\frac{\bar{E}_{\gamma}\left(E_{\gamma}+\bar{E}_{\gamma}\right)}{m_{e}^{2}}
$$

and $\Theta\left(\left\{E_{i} ; T\right\}\right)$ is zero unless $B \geq 1$ and

$$
\frac{E_{\gamma}+\bar{E}_{\gamma}}{2}(1-\sqrt{1-1 / B}) \leq E_{e} \leq \frac{E_{\gamma}+\bar{E}_{\gamma}}{2}(1+\sqrt{1-1 / B})
$$

These inequalities reflect kinematic constraints on the electron and positron energies. ${ }^{4}$

\footnotetext{
${ }^{3}$ This than ref. [86] by a factor of two to account each collision producing an electron and a positron.

${ }^{4}$ These limits were not specified explicitly in ref. [40].
} 


\section{A.2 Rates for Inverse Compton scattering (IC)}

Inverse Compton (IC) scattering in this context corresponds to the scattering of high energy electrons (or positrons) off CMB photons, $e^{ \pm}+\gamma_{\mathrm{BG}} \rightarrow e^{ \pm}+\gamma$. This reduces the electron energy and produces an energetic photon. We evaluate the corresponding contributions to $\Gamma_{e}, K_{e e}$, and $K_{\gamma e}$.

\section{A.2.1 IC electron relaxation}

The relaxation rate for electrons due to IC was computed in ref. [87] and reproduced in ref. [40]. For $e\left(E_{e}\right)+\gamma\left(\bar{E}_{\gamma}\right) \rightarrow e\left(E_{e}^{\prime}\right)+\gamma\left(E_{\gamma}\right)$, the expression is

$$
\Delta \Gamma_{e}\left(E_{e}\right)=\frac{2}{\pi} \alpha^{2} \frac{1}{E_{e}^{2}} \int_{0}^{E_{e}} d E_{\gamma} \int_{0}^{\infty} d \bar{E}_{\gamma} \bar{E}_{\gamma}\left(e^{\bar{E}_{\gamma} / T}-1\right)^{-1} F\left(E_{\gamma}, E_{e}, \bar{E}_{\gamma}\right),
$$

where $^{5}$

$$
F\left(E_{\gamma}, E_{e}, \bar{E}_{\gamma}\right)= \begin{cases}2 q \ln q+(1+2 q)(1-q)+\frac{(\xi q)^{2}(1-q)}{2(1+\xi q)} ; q \in[0,1] \\ 0 & ; \text { otherwise }\end{cases}
$$

with

$$
\xi=\frac{4 \bar{E}_{\gamma} E_{e}}{m_{e}^{2}}, \quad q=\frac{E_{\gamma}}{\xi\left(E_{e}-E_{\gamma}\right)} .
$$

Note that this expression is based on an approximation that is only valid for $\bar{E}_{\gamma}<E_{\gamma}$ and $q \geq\left(m_{e} / 2 E_{e}\right)^{2}$ [87]. However, for the energies and temperatures relevant for photodissociation, we find that applying $q \geq 0$ is a good approximation. The IC electron relaxation rate can be written in the simpler form

$$
\Delta \Gamma_{e}\left(E_{e}\right)=\frac{2}{\pi} \alpha^{2}\left(\frac{T}{m_{e}}\right)^{3} m_{e} \frac{1}{y_{e}^{2}} \mathcal{H}\left(y_{e}\right),
$$

with

$$
\begin{aligned}
\frac{1}{y_{e}^{2}} \mathcal{H}\left(y_{e}\right) & =\int_{0}^{y_{e}} d y_{\gamma} \int_{0}^{\infty} d y y\left(e^{y}-1\right)^{-1} F\left(E_{\gamma}, E_{e}, \bar{E}_{\gamma}\right) \\
& \simeq 3.07 /\left(1+12 y_{e}+y_{e}^{2}\right)^{0.47},
\end{aligned}
$$

where the second line is a numerical fit valid to within about $5 \%$ over $y_{e} \in\left[10^{-5}, 10^{2}\right]$. For $y_{e} \ll 1$ this coincides with IC in the Thomson limit: $\Delta \Gamma_{e}=\sigma_{T} n_{\gamma}(T)$, where $\sigma_{T}=$ $(8 \pi / 3) \alpha^{2} / m_{e}^{2}$ is the Thomson cross section and $n_{\gamma}$ is the photon density.

\section{A.2.2 IC transfer to electrons}

The IC process also produces an electron with a lower energy than the initial value. The corresponding transfer kernel for $e\left(E_{e}^{\prime}\right)+\gamma\left(\bar{E}_{\gamma}\right) \rightarrow e\left(E_{e}\right)+\gamma\left(E_{\gamma}\right)$ is

$$
\Delta K_{e e}\left(E_{e}, E_{e}^{\prime}\right)=\frac{2}{\pi} \alpha^{2} \frac{1}{E_{e}^{\prime 2}} \int_{0}^{\infty} d \bar{E}_{\gamma} \bar{E}_{\gamma}\left(e^{-\bar{E}_{\gamma} / T}-1\right)^{-1} F\left(E_{\gamma}, E_{e}^{\prime}, \bar{E}_{\gamma}\right),
$$

\footnotetext{
${ }^{5}$ The integration over $E_{\gamma}$ in eq. (A.9) covers the interval $\left[0, E_{e}\right]$, and not $\left[E_{e}, \infty\right)$ as given in ref. [40].
} 
where $E_{e}^{\prime}>E_{e}$,

$$
E_{\gamma}=E_{e}^{\prime}+\bar{E}_{\gamma}-E_{e},
$$

and the function $F\left(E_{\gamma}, E_{e}^{\prime}, \bar{E}_{\gamma}\right)$ is given by eq. (A.10).

\section{A.2.3 IC transfer to photons}

For photon energy transfer via $e\left(E_{e}\right)+\gamma\left(\bar{E}_{\gamma}\right) \rightarrow e\left(E_{e}^{\prime}\right)+\gamma\left(E_{\gamma}\right)$, the kernel is ${ }^{6}$

$$
\Delta K_{\gamma e}\left(E_{\gamma}, E_{e}\right)=\frac{2}{\pi} \alpha^{2} \frac{1}{E_{e}^{2}} \int_{0}^{\infty} d \bar{E}_{\gamma} \bar{E}_{\gamma}\left(e^{-\bar{E}_{\gamma} / T}-1\right)^{-1} F\left(E_{\gamma}, E_{e}, \bar{E}_{\gamma}\right)
$$

where $F\left(\left\{E_{i}\right\}\right)$ is defined in eq. (A.10).

\section{A.3 Photon Photon Scattering (PP)}

Photon photon (PP) scattering contributes to photon relaxation and transfer.

\section{A.3.1 PP photon relaxation}

The contribution to the relaxation rate is approximately $[52,88]$

$$
\Delta \Gamma_{\gamma}\left(E_{\gamma}\right) \simeq(0.1513) \alpha^{4} m_{e}\left(\frac{T}{m_{e}}\right)^{3} y_{\gamma}^{3} e^{-y_{\gamma}},
$$

with $y_{\gamma}=E_{\gamma} T / m_{e}^{2}$. Note that this form only applies for $y_{\gamma}<1$, and thus we add an exponential by hand to provide a smooth cutoff at larger values.

\section{A.3.2 PP photon transfer}

For transfer $\gamma\left(E_{\gamma}^{\prime}\right)+\gamma_{\mathrm{BG}} \rightarrow \gamma\left(E_{\gamma}\right)+\gamma$, the kernel is $[52,88]$

$$
\Delta K_{\gamma \gamma}\left(E_{\gamma}, E_{\gamma}^{\prime}\right) \simeq(0.4324) \alpha^{4}\left(\frac{T}{m_{e}}\right)^{4} y_{\gamma}^{\prime 2}\left(1-\frac{y_{\gamma}}{y_{\gamma}^{\prime}}+\frac{y_{\gamma}^{2}}{y_{\gamma}^{\prime 2}}\right)^{2} e^{-y_{\gamma}}
$$

with $E_{\gamma}<E_{\gamma}^{\prime}$ and an exponential has again been added to cut off this form when $y_{\gamma}>1$.

\section{A.4 Pair Creation on Nuclei (PCN)}

Photon scattering on background nuclei can create $e^{+} e^{-}$pairs, $\gamma\left(E_{\gamma}\right)+N \rightarrow N+e^{+}+e^{-}$. This reduces the photon energy and injects energy into electrons and positrons.

\section{A.4.1 PCN photon relaxation}

The contribution to photon relaxation is [40]

$$
\Delta \Gamma_{\gamma}\left(E_{\gamma}\right)=\sum_{Z} n_{Z} \sigma_{\mathrm{PCN}}^{(Z)}\left(E_{\gamma}\right)
$$

\footnotetext{
${ }^{6}$ The corresponding expression in ref. [40] appears to have a typo by a factor of two.
} 
where the sum runs over $Z=\mathrm{H},{ }^{4} \mathrm{He}$ and $n_{Z}$ are their corresponding densities. The cross section for $x=2 E_{\gamma} / m_{e} \geq 8$ is

$$
\begin{aligned}
\sigma_{\mathrm{PCN}}^{(Z)}= & Z^{2} \frac{\alpha^{3}}{m_{e}^{2}}\left(\frac{28}{9} \ln x-\frac{218}{27}\right. \\
& \left.+\left(\frac{4}{x}\right)^{2}\left[\frac{2}{3}(\ln x)^{3}-(\ln x)^{2}+\left(6-\frac{\pi^{2}}{3}\right) \ln x+2 \zeta(3)+\frac{\pi^{2}}{6}-\frac{7}{2}\right]\right)+\ldots
\end{aligned}
$$

while for $4 \leq x<8$ it is

$$
\sigma_{\mathrm{PCN}}^{(Z)}=Z^{2} \frac{2 \pi}{3} \frac{\alpha^{3}}{m_{e}^{2}}\left(\frac{x-4}{x}\right)^{3}\left(1+\frac{1}{2} \rho+\frac{23}{40} \rho^{2}+\frac{11}{60} \rho^{3}+\frac{29}{960} \rho^{4}+\ldots\right),
$$

with

$$
\rho=\frac{x-4}{2+2 \sqrt{x}+x / 2} .
$$

Note that $x=4$ is the threshold for the process to occur.

\section{A.4.2 PCN electron transfer}

The contribution to the electron transfer kernel is [40]

$$
\Delta K_{e \gamma}\left(E_{e}, E_{\gamma}\right)=\sum_{Z} n_{Z} \frac{d \sigma_{\mathrm{PCN}}^{(Z)}}{d E_{e}}
$$

with

$$
\begin{aligned}
\frac{d \sigma_{\mathrm{PCN}}^{(Z)}}{d E_{e}}=Z^{2} & \frac{\alpha^{3}}{m_{e}^{2}}\left(\frac{p p^{\prime}}{E_{\gamma}^{3}}\right)\left(-\frac{4}{3}-2 E_{e} E_{e}^{\prime} \frac{p^{2}+p^{\prime 2}}{p^{2} p^{\prime 2}}\right. \\
& +m_{e}^{2}\left(\frac{\ell^{\prime} E_{e}}{p^{\prime 3}}+\frac{\ell E_{e}^{\prime}}{p^{3}}-\frac{\ell \ell^{\prime}}{p p^{\prime}}\right) \\
& +L\left[-\frac{8 E_{e} E_{e}^{\prime}}{3 p p^{\prime}}+\frac{E_{\gamma}^{2}}{p^{3} p^{\prime 3}}\left(E_{e}^{2} E_{e}^{\prime 2}+p^{2} p^{\prime 2}-m_{e}^{2} E_{e} E_{e}^{\prime}\right)\right. \\
& \left.\left.-\frac{m_{e}^{2} E_{\gamma}}{2 p p^{\prime}}\left(\ell \frac{E_{e} E_{e}^{\prime}-p^{2}}{p^{3}}+\ell^{\prime} \frac{E_{e} E_{e}^{\prime}-p^{\prime 2}}{p^{\prime 3}}\right)\right]\right)
\end{aligned}
$$

where $E_{e}^{\prime}=\left(E_{\gamma}-E_{e}\right), p^{\left({ }^{\prime}\right)}=\sqrt{E_{e}^{\left({ }^{\prime}\right) 2}-m_{e}^{2}}$, and

$$
\begin{aligned}
\ell^{\left({ }^{\prime}\right)} & =\ln \left(\frac{E_{e}^{\left({ }^{\prime}\right)}+p^{\left({ }^{\prime}\right)}}{E_{e}^{(\prime)}-p^{\left({ }^{\prime}\right)}}\right), \\
L & =\ln \left(\frac{E_{e} E_{e}^{\prime}+p p^{\prime}+m_{e}^{2}}{E_{e} E_{e}^{\prime}-p p^{\prime}+m_{e}^{2}}\right) .
\end{aligned}
$$

As before, the relevant nuclei are $\mathrm{H}$ and ${ }^{4} \mathrm{He}$. 


\section{A.5 Compton Scattering (CS)}

Compton scattering in this context refers to high-energy photons colliding with background electrons, $\gamma+e_{\mathrm{BG}}^{-} \rightarrow \gamma+e^{-}$. This reduces the photon energy and transfers it to the scattered electron.

\section{A.5.1 CS photon relaxation}

The contribution to the relaxation rate is

$$
\Delta \Gamma_{\gamma}\left(E_{\gamma}\right)=n_{e} \sigma_{\mathrm{CS}}
$$

where $n_{e}$ is the background electron density given and

$$
\sigma_{\mathrm{CS}}\left(E_{\gamma}\right)=2 \pi \frac{\alpha^{2}}{m_{e}^{2}} \frac{1}{x}\left[\left(1-\frac{4}{x}-\frac{8}{x^{2}}\right) \ln (1+x)+\frac{1}{2}+\frac{8}{x}-\frac{1}{2(1+x)^{2}}\right],
$$

with $x=2 E_{\gamma} / m_{e}$.

\section{A.5.2 CS photon transfer}

The energy of the initial CS photon $E_{\gamma}^{\prime}$ is partially transferred to the energy $E_{\gamma}$ of the outgoing photon. This contributes to the transfer kernel by [40, 52, 75]

$$
\Delta K_{\gamma \gamma}\left(E_{\gamma}, E_{\gamma}^{\prime}\right)=n_{e} \frac{d \sigma_{\mathrm{CS}}\left(E_{\gamma}, E_{\gamma}^{\prime}\right)}{d E_{\gamma}}
$$

with

$$
\frac{d \sigma_{\mathrm{CS}}\left(E_{\gamma}, E_{\gamma}^{\prime}\right)}{d E_{\gamma}}=\pi \frac{\alpha^{2}}{m_{e}} \frac{1}{E_{\gamma}^{\prime 2}}\left[\frac{E_{\gamma}^{\prime}}{E_{\gamma}}+\frac{E_{\gamma}}{E_{\gamma}^{\prime}}+\left(\frac{m_{e}}{E_{\gamma}}-\frac{m_{e}}{E_{\gamma}^{\prime}}\right)^{2}-2 m_{e}\left(\frac{1}{E_{\gamma}}-\frac{1}{E_{\gamma}^{\prime}}\right)\right] .
$$

This expression is valid only for $E_{\gamma}^{\prime} /\left(1+2 E_{\gamma}^{\prime} / m_{e}\right) \leq E_{\gamma} \leq E_{\gamma}^{\prime}$ and zero otherwise. ${ }^{7}$

\section{A.5.3 CS electron transfer}

Energy transfer to electrons by CS is given by

$$
\Delta K_{e \gamma}\left(E_{e}, E_{\gamma}^{\prime}\right)=n_{e} \frac{d \sigma_{\mathrm{CS}}\left(E_{\gamma}, E_{\gamma}^{\prime}\right)}{d E_{\gamma}},
$$

where $E_{\gamma}=\left(E_{\gamma}^{\prime}+m_{e}-E_{e}\right), E_{\gamma}^{\prime} \geq E_{e}$, and the differential cross section is given in eq. (A.31).

Open Access. This article is distributed under the terms of the Creative Commons Attribution License (CC-BY 4.0), which permits any use, distribution and reproduction in any medium, provided the original author(s) and source are credited.

\footnotetext{
${ }^{7}$ The differential CS expression in ref. [52] appears to have an incorrect sign in the last term of eq. (A.31), and neither refs. [40, 52] give the lower bound on $E_{\gamma}$ stated explicitly in ref. [75].
} 


\section{References}

[1] S. Sarkar, Big bang nucleosynthesis and physics beyond the standard model, Rept. Prog. Phys. 59 (1996) 1493 [hep-ph/9602260] [INSPIRE].

[2] F. Iocco et al., Primordial nucleosynthesis: from precision cosmology to fundamental physics, Phys. Rept. 472 (2009) 1 [arXiv:0809.0631] [InSPIRE].

[3] K. Jedamzik and M. Pospelov, Big Bang nucleosynthesis and particle dark matter, New J. Phys. 11 (2009) 105028 [arXiv: 0906. 2087] [INSPIRE].

[4] M. Pospelov and J. Pradler, Big Bang nucleosynthesis as a probe of new physics, Ann. Rev. Nucl. Part. Sci. 60 (2010) 539 [arXiv:1011.1054].

[5] D.N. Schramm and R.V. Wagoner, Element production in the early universe, Ann. Rev. Nucl. Part. Sci. 27 (1977) 37.

[6] J. Bernstein, L.S. Brown and G. Feinberg, Cosmological Helium production simplified, Rev. Mod. Phys. 61 (1989) 25 [INSPIRE].

[7] T.P. Walker et al., Primordial nucleosynthesis redux, Astrophys. J. 376 (1991) 51 [InSPIRE].

[8] R.H. Cyburt, B.D. Fields, K.A. Olive and T.-H. Yeh, Big Bang nucleosynthesis: 2015, Rev. Mod. Phys. 88 (2016) 015004 [arXiv: 1505.01076] [INSPIRE].

[9] M. Asplund et al., Lithium isotopic abundances in metal-poor halo stars, Astrophys. J. 644 (2006) 229 [astro-ph/0510636] [INSPIRE].

[10] L. Sbordone et al., The metal-poor end of the Spite plateau. 1: stellar parameters, metallicities and lithium abundances, Astron. Astrophys. 522 (2010) A26 [arXiv:1003.4510] [INSPIRE].

[11] R.H. Cyburt, B.D. Fields and K.A. Olive, An update on the big bang nucleosynthesis prediction for Li-\%: the problem worsens, JCAP 11 (2008) 012 [arXiv:0808.2818] [INSPIRE].

[12] B.D. Fields, The primordial lithium problem, Ann. Rev. Nucl. Part. Sci. 61 (2011) 47 [arXiv: 1203.3551] [INSPIRE].

[13] M. Kawasaki, K. Kohri and N. Sugiyama, Cosmological constraints on late time entropy production, Phys. Rev. Lett. 82 (1999) 4168 [astro-ph/9811437] [INSPIRE].

[14] M. Kawasaki, K. Kohri and N. Sugiyama, MeV scale reheating temperature and thermalization of neutrino background, Phys. Rev. D 62 (2000) 023506 [astro-ph/0002127] [INSPIRE].

[15] S. Hannestad, What is the lowest possible reheating temperature?, Phys. Rev. D 70 (2004) 043506 [astro-ph/0403291] [INSPIRE].

[16] Planck collaboration, N. Aghanim et al., Planck 2018 results. VI. Cosmological parameters, arXiv: 1807.06209 [INSPIRE].

[17] J.R. Ellis, D.V. Nanopoulos and S. Sarkar, The cosmology of decaying gravitinos, Nucl. Phys. B 259 (1985) 175 [INSPIRE].

[18] R. Juszkiewicz, J. Silk and A. Stebbins, Constraints on cosmologically regenerated gravitinos, Phys. Lett. B 158 (1985) 463.

[19] S. Dimopoulos, R. Esmailzadeh, L.J. Hall and G.D. Starkman, Is the universe closed by baryons? Nucleosynthesis with a late decaying massive particle, Astrophys. J. 330 (1988) 545 [INSPIRE]. 
[20] M.H. Reno and D. Seckel, Primordial nucleosynthesis: the effects of injecting hadrons, Phys. Rev. D 37 (1988) 3441 [INSPIRE].

[21] S. Dimopoulos, R. Esmailzadeh, L.J. Hall and G.D. Starkman, Limits on late decaying particles from nucleosynthesis, Nucl. Phys. B 311 (1989) 699 [INSPIRE].

[22] J.R. Ellis et al., Astrophysical constraints on massive unstable neutral relic particles, Nucl. Phys. B 373 (1992) 399 [INSPIRE].

[23] T. Moroi, H. Murayama and M. Yamaguchi, Cosmological constraints on the light stable gravitino, Phys. Lett. B 303 (1993) 289 [InSPIRE].

[24] M. Kawasaki and T. Moroi, Gravitino production in the inflationary universe and the effects on big bang nucleosynthesis, Prog. Theor. Phys. 93 (1995) 879 [hep-ph/9403364] [InSPIRE].

[25] R.H. Cyburt, J.R. Ellis, B.D. Fields and K.A. Olive, Updated nucleosynthesis constraints on unstable relic particles, Phys. Rev. D 67 (2003) 103521 [astro-ph/0211258] [INSPIRE].

[26] K. Jedamzik, Did something decay, evaporate, or annihilate during Big Bang nucleosynthesis?, Phys. Rev. D 70 (2004) 063524 [astro-ph/0402344] [InSPIRE].

[27] M. Kawasaki, K. Kohri and T. Moroi, Big-Bang nucleosynthesis and hadronic decay of long-lived massive particles, Phys. Rev. D 71 (2005) 083502 [astro-ph/0408426] [INSPIRE].

[28] K. Jedamzik, Big bang nucleosynthesis constraints on hadronically and electromagnetically decaying relic neutral particles, Phys. Rev. D 74 (2006) 103509 [hep-ph/0604251] [INSPIRE].

[29] M. Kawasaki, K. Kohri, T. Moroi and A. Yotsuyanagi, Big-Bang nucleosynthesis and gravitino, Phys. Rev. D 78 (2008) 065011 [arXiv:0804.3745] [INSPIRE].

[30] M. Kawasaki, K. Kohri, T. Moroi and Y. Takaesu, Revisiting Big-Bang nucleosynthesis constraints on long-lived decaying particles, Phys. Rev. D 97 (2018) 023502 [arXiv: 1709.01211] [INSPIRE].

[31] J.A. Frieman, E.W. Kolb and M.S. Turner, Eternal annihilations: new constraints on longlived particles from Big Bang nucleosynthesis, Phys. Rev. D 41 (1990) 3080 [INSPIRE].

[32] J. Hisano, M. Kawasaki, K. Kohri and K. Nakayama, Positron/gamma-ray signatures of dark matter annihilation and Big-Bang nucleosynthesis, Phys. Rev. D 79 (2009) 063514 [Erratum ibid. D 80 (2009) 029907] [arXiv:0810.1892] [INSPIRE].

[33] J. Hisano et al., Cosmic rays from dark matter annihilation and Big-Bang nucleosynthesis, Phys. Rev. D 79 (2009) 083522 [arXiv:0901.3582] [InSPIRE].

[34] M. Kawasaki, K. Kohri, T. Moroi and Y. Takaesu, Revisiting Big-Bang nucleosynthesis constraints on dark-matter annihilation, Phys. Lett. B 751 (2015) 246 [arXiv:1509.03665] [INSPIRE].

[35] R.H. Cyburt, B.D. Fields, K.A. Olive and E. Skillman, New BBN limits on physics beyond the standard model from ${ }^{4} H e$, Astropart. Phys. 23 (2005) 313 [astro-ph/0408033] [INSPIRE].

[36] C.M. Ho and R.J. Scherrer, Limits on MeV dark matter from the effective number of neutrinos, Phys. Rev. D 87 (2013) 023505 [arXiv: 1208.4347] [INSPIRE].

[37] C. Boehm, M.J. Dolan and C. McCabe, A lower bound on the mass of cold thermal dark matter from Planck, JCAP 08 (2013) 041 [arXiv: 1303.6270] [INSPIRE].

[38] K.M. Nollett and G. Steigman, BBN and the CMB constrain light, electromagnetically coupled WIMPs, Phys. Rev. D 89 (2014) 083508 [arXiv:1312.5725] [INSPIRE]. 
[39] R.J. Protheroe, T. Stanev and V.S. Berezinsky, Electromagnetic cascades and cascade nucleosynthesis in the early universe, Phys. Rev. D 51 (1995) 4134 [astro-ph/9409004] [INSPIRE].

[40] M. Kawasaki and T. Moroi, Electromagnetic cascade in the early universe and its application to the big bang nucleosynthesis, Astrophys. J. 452 (1995) 506 [astro-ph/9412055] [INSPIRE].

[41] A. Fradette, M. Pospelov, J. Pradler and A. Ritz, Cosmological constraints on very dark photons, Phys. Rev. D 90 (2014) 035022 [arXiv: 1407.0993] [INSPIRE].

[42] J. Berger, K. Jedamzik and D.G.E. Walker, Cosmological constraints on decoupled dark photons and dark Higgs, JCAP 11 (2016) 032 [arXiv:1605.07195] [INSPIRE].

[43] A. Fradette and M. Pospelov, BBN for the LHC: constraints on lifetimes of the Higgs portal scalars, Phys. Rev. D 96 (2017) 075033 [arXiv:1706.01920] [INSPIRE].

[44] A. Soni and Y. Zhang, Hidden SU(N) glueball dark matter, Phys. Rev. D 93 (2016) 115025 [arXiv: 1602.00714] [INSPIRE].

[45] L. Forestell, D.E. Morrissey and K. Sigurdson, Non-abelian dark forces and the relic densities of dark glueballs, Phys. Rev. D 95 (2017) 015032 [arXiv:1605.08048] [INSPIRE].

[46] L. Forestell, D.E. Morrissey and K. Sigurdson, Cosmological bounds on non-abelian dark forces, Phys. Rev. D 97 (2018) 075029 [arXiv:1710.06447] [InSPIRE].

[47] B. Henning and H. Murayama, Constraints on light dark matter from Big Bang nucleosynthesis, arXiv:1205.6479 [INSPIRE].

[48] Y. Hochberg et al., SIMPs through the axion portal, arXiv:1806.10139 [INSPIRE].

[49] S. Sarkar and A.M. Cooper-Sarkar, Cosmological and experimental constraints on the tau neutrino, Phys. Lett. B 148 (1984) 347.

[50] H. Ishida, M. Kusakabe and H. Okada, Effects of long-lived 10 MeV-scale sterile neutrinos on primordial elemental abundances and the effective neutrino number, Phys. Rev. D 90 (2014) 083519 [arXiv: 1403.5995] [INSPIRE].

[51] V. Poulin and P.D. Serpico, Loophole to the Universal Photon Spectrum in Electromagnetic Cascades and Application to the Cosmological Lithium Problem, Phys. Rev. Lett. 114 (2015) 091101 [arXiv: 1502.01250] [INSPIRE].

[52] V. Poulin and P.D. Serpico, Nonuniversal BBN bounds on electromagnetically decaying particles, Phys. Rev. D 91 (2015) 103007 [arXiv:1503.04852] [INSPIRE].

[53] G.R. Blumenthal and R.J. Gould, Bremsstrahlung, synchrotron radiation and compton scattering of high-energy electrons traversing dilute gases, Rev. Mod. Phys. 42 (1970) 237 [INSPIRE].

[54] A. Birkedal, K.T. Matchev, M. Perelstein and A. Spray, Robust $\gamma$ ray signature of WIMP dark matter, hep-ph/0507194 [INSPIRE].

[55] J. Mardon, Y. Nomura, D. Stolarski and J. Thaler, Dark matter signals from cascade annihilations, JCAP 05 (2009) 016 [arXiv:0901.2926] [INSPIRE].

[56] R. Evans, The atomic nucleus, Krieger publishing company, U.S.A. (2003).

[57] A.N. Gorbunov and A.T. Varfolomeev, Cross sections of the reactions ${ }^{3} H e(\gamma, p)$ D2 and ${ }^{3} H e$ $(\gamma, n) 2 p$, Phys. Lett. 11 (1964) 137.

[58] R. Pfeiffer, Der Kernphotoeffekt am ${ }^{3}$ H, Z. Phys. 208 (1968) 129. 
[59] D.D. Faul, B.L. Berman, P. Meyer and D.L. Olson, Photodisintegration of ${ }^{3} H$, Phys. Rev. Lett. 44 (1980) 129 [INSPIRE].

[60] Yu.M. Arkatov et al., Photodisintegration of He-4 nucleus down to threshold of meson production, Ukr. Fiz. Zh.(Russ. Ed.) 23 (1978) 1818.

[61] J. D. Irish et al., Photoneutron Angular Distributions for ${ }^{4}$ He, Can. J. Phys. 53 (1975) 802.

[62] C.K. Malcom, D.V. Webb, Y.M. Shin and D.M. Skopik, Evidence of a $2+$ state from the ${ }^{4} \mathrm{He}(\gamma, n){ }^{3}$ He reaction, Phys. Lett. B 47 (1973) 433.

[63] O. Pisanti et al., PArthENoPE: public algorithm evaluating the nucleosynthesis of primordial elements, Comput. Phys. Commun. 178 (2008) 956 [arXiv:0705.0290] [INSPIRE].

[64] R. Consiglio et al., PArthENoPE reloaded, Comput. Phys. Commun. 233 (2018) 237 [arXiv:1712.04378] [INSPIRE].

[65] E. Aver, K.A. Olive and E.D. Skillman, The effects of He I $\lambda 10830$ on helium abundance determinations, JCAP 07 (2015) 011 [arXiv: 1503.08146] [INSPIRE].

[66] R.J. Cooke, M. Pettini and C.C. Steidel, One percent determination of the primordial deuterium abundance, Astrophys. J. 855 (2018) 102 [arXiv:1710.11129] [INSPIRE].

[67] J. Geiss and G. Gloeckler, Isotopic composition of H, He and Ne in the protosolar cloud, Space Sci. Rev. 106 (2003) 3.

[68] A. Peimbert, M. Peimbert and V. Luridiana, The primordial helium abundance and the number of neutrino families, Rev. Mex. Astron. Astrofis. 52 (2016) 419 [arXiv: 1608.02062] [INSPIRE].

[69] Y.I. Izotov, T.X. Thuan and N.G. Guseva, A new determination of the primordial He abundance using the HeI $\lambda 10830$ A emission line: cosmological implications, Mon. Not. Roy. Astron. Soc. 445 (2014) 778 [arXiv:1408.6953] [INSPIRE].

[70] L.E. Marcucci, G. Mangano, A. Kievsky and M. Viviani, Implication of the proton-deuteron radiative capture for Big Bang nucleosynthesis, Phys. Rev. Lett. 116 (2016) 102501 [Erratum ibid. 117 (2016) 049901] [arXiv: 1510.07877] [INSPIRE].

[71] J.A. Adams, S. Sarkar and D.W. Sciama, CMB anisotropy in the decaying neutrino cosmology, Mon. Not. Roy. Astron. Soc. 301 (1998) 210 [astro-ph/9805108] [INSPIRE].

[72] X.-L. Chen and M. Kamionkowski, Particle decays during the cosmic dark ages, Phys. Rev. D 70 (2004) 043502 [astro-ph/0310473] [INSPIRE].

[73] N. Padmanabhan and D.P. Finkbeiner, Detecting dark matter annihilation with CMB polarization: Signatures and experimental prospects, Phys. Rev. D 72 (2005) 023508 [astro-ph/0503486] [INSPIRE].

[74] L. Zhang et al., Constraints on radiative dark-matter decay from the cosmic microwave background, Phys. Rev. D 76 (2007) 061301 [arXiv:0704.2444] [INSPIRE].

[75] T.R. Slatyer, N. Padmanabhan and D.P. Finkbeiner, CMB constraints on WIMP annihilation: energy absorption during the recombination epoch, Phys. Rev. D 80 (2009) 043526 [arXiv:0906.1197] [INSPIRE].

[76] J.M. Cline and P. Scott, Dark matter CMB constraints and likelihoods for poor particle physicists, JCAP 03 (2013) 044 [Erratum ibid. 05 (2013) E01] [arXiv:1301.5908] [INSPIRE].

[77] J.L. Feng, A. Rajaraman and F. Takayama, SuperWIMP dark matter signals from the early universe, Phys. Rev. D 68 (2003) 063504 [hep-ph/0306024] [INSPIRE]. 
[78] M. Kaplinghat and M.S. Turner, Precision cosmology and the density of baryons in the universe, Phys. Rev. Lett. 86 (2001) 385 [astro-ph/0007454] [INSPIRE].

[79] W. Hu and J. Silk, Thermalization and spectral distortions of the cosmic background radiation, Phys. Rev. D 48 (1993) 485 [InSPIRE].

[80] W. Hu and J. Silk, Thermalization constraints and spectral distortions for massive unstable relic particles, Phys. Rev. Lett. 70 (1993) 2661 [INSPIRE].

[81] D.J. Fixsen et al., The Cosmic Microwave Background spectrum from the full COBE FIRAS data set, Astrophys. J. 473 (1996) 576 [astro-ph/9605054] [INSPIRE].

[82] J. Chluba and R.A. Sunyaev, The evolution of CMB spectral distortions in the early Universe, Mon. Not. Roy. Astron. Soc. 419 (2012) 1294 [arXiv:1109.6552].

[83] A. Kogut et al., The Primordial Inflation Explorer (PIXIE): a nulling polarimeter for Cosmic Microwave Background observations, JCAP 07 (2011) 025 [arXiv:1105.2044] [INSPIRE].

[84] M. Kusakabe, A.B. Balantekin, T. Kajino and Y. Pehlivan, Big-bang nucleosynthesis limit on the neutral fermion decays into neutrinos, Phys. Rev. D 87 (2013) 085045 [arXiv:1303.2291] [INSPIRE].

[85] M. Hufnagel, K. Schmidt-Hoberg and S. Wild, BBN constraints on MeV-scale dark sectors. Part II. Electromagnetic decays, JCAP 11 (2018) 032 [arXiv: 1808.09324] [INSPIRE].

[86] F.A. Aharonian, A.M. Atoian and A.M. Nagapetian, Photoproduction of electron-positron pairs in compact X-ray sources, Astrofizika 19 (1983) 323.

[87] F.C. Jones, Calculated spectrum of inverse-Compton-scattered photons, Phys. Rev. 167 (1968) 1159 [INSPIRE].

[88] R. Svensson and A.A. Zdziarski, Photon-photon scattering of gamma rays at cosmological distances, Astrophys. J. 349 (1990) 415 [INSPIRE]. 